\title{
Accurate description of heterogeneous tumors for biologically optimized radiation therapy
}

\author{
Johan Nilsson
}

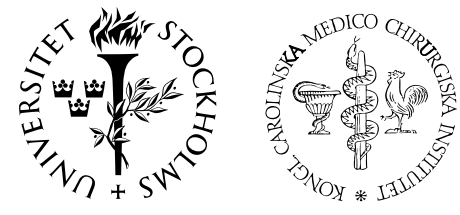

Division of Medical Radiation Physics

Department of Oncology-Pathology

Stockholm University and Karolinska Institutet Stockholm, Sweden

December 2004 


\begin{abstract}
In this thesis, a model of tissue oxygenation is presented, that takes into account the heterogeneous nature of tumor vasculature. Even though the model is rather simple, the resulting oxygen distributions agree very well with clinically observed oxygen distributions for most tumors and healthy normal tissues. The model shows that the vascular density may not describe the oxygenation of a tissue sufficiently well, unless the heterogeneity of the vascular system is taken into account. Based on the oxygen distributions from the tissue model, the associated radiation response at low and high doses can be determined.

The radiation response of heterogeneous tumors should preferably be described by two clonogen compartments, one resistant and one sensitive, dominating the response at high and low radiation doses, respectively. Furthermore, each compartment should be characterized by the effective radiation resistance and the effective clonogen number. The resistant-sensitive model of radiation response has been analyzed in great detail. It accurately describes the response of severely heterogeneous tumors, both at low and high doses and LET values. The effective response parameters are given as integrals, averaged over the whole spectrum of radiation resistance. The parameters can also be determined from clinically established dose-response relations.

The main properties of the dose-response relation for a generally heterogeneous tumor is described in some detail. The normalized dose-response gradient has been generalized to take heterogeneities in both dose delivery and radiation response into account. This quantity is important for accurate treatment plan optimization using intensity modulated radiation therapy for individual patients.
\end{abstract}

(C) 2004 Johan Nilsson (pp 1-39, paper IV)

ISBN 91-628-6362-2 


\section{List of publications}

This thesis is based on the following publications, which are referred to in the text by their Roman numerals.

I Lind, B. K., Nilsson J., Löf, J. and Brahme A., 2001, Generalization of the normalized dose-response gradient to non-uniform dose delivery. Acta Oncologica, 40, 718-724

II Brahme A., Nilsson J., Belkić, Dž., 2001, Biologically optimized radiation therapy. Acta Oncologica, 40, 725-734

III Nilsson, J., Lind, B. K. and Brahme, A., 2002, Radiation response of hypoxic and generally heterogeneous tissues. International Journal of Radiation Biology, 78, 389-405

IV Nilsson, J., Lind, B. K. and Brahme, A., 2004, Accurate description of heterogeneous tumors by effective radiation-sensitive and resistant cell compartments (Manuscript) 


\section{Contents}

Abstract ii

List of publications iii

1. Introduction 1

1.1. Tumor response heterogeneities in radiation therapy . . . . . . . . 1

1.1.1. Micro- and macroscopic tumor response heterogeneities . . . 1

1.1.2. The radiation response of hypoxic cells and tumors . . . . . 3

2. Tissue oxygenation modeling 5

2.1. Overview of existing models of microvascular networks . . . . . . . 5

2.2. A tissue oxygenation model including vascular heterogeneity . . . . 6

3. The radiation response of heterogeneous tumors 8

3.1. Basic cell survival . . . . . . . . . . . . . . . . . . . 8

3.2. Heterogeneous radiation response based on binomial and Poisson statis-

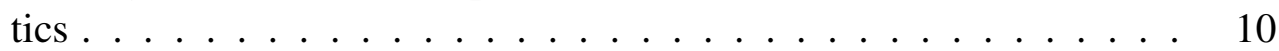

3.3. Generalized cell survival . . . . . . . . . . . . . . . . . . 13

3.4. The effective radiation response . . . . . . . . . . . . 15

3.4.1. Effective exponential response . . . . . . . . . . . . . 15

3.5. The generalized dose-response gradient . . . . . . . . . . . 17

3.6. The resistant-sensitive (r-s) cell survival model . . . . . . . . . . 18

3.6.1. Reduction of the r-s model into one component . . . . . . 18

3.6.2. The effective radiation response when the resistant or sensitive clonogens dominate . . . . . . . . . . . . . . . . . 19

3.6.3. The resistant fraction . . . . . . . . . . . . . . . . 20

3.7. The frequency function of $\boldsymbol{D}_{0}$ and the mean and effective values of

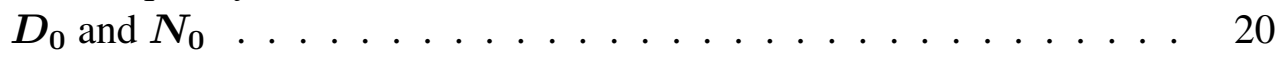

3.8. Incorporating tumor dynamics in constant-dose fractionation modeling 22

3.8.1. No reoxygenation . . . . . . . . . . . . . . . . . 22

3.8.2. Reoxygenation . . . . . . . . . . . . . . . . . . . 24

3.8.3. Repopulation . . . . . . . . . . . . . . . . . . 25

3.9. Oxygen dependence of the cell survival model parameters . . . . . . 25 
3.10. The response of hypoxic tumors to beams of low and high ionization density ......................... 27

$\begin{array}{lr}\text { 4. Conclusions } & 28\end{array}$

A. Appendix $\quad 29$

A.1. Effective response parameters for some survival functions . . . . . . . 29

A.1.1. The LQ model . . . . . . . . . . . . . . . . . . . 29

A.1.2. The RCR model . . . . . . . . . . . . . . . . . 30

A.2. The effective radiation response in terms of $\boldsymbol{D}_{\mathbf{0} \text {,eff }}$ and $\boldsymbol{N}_{\mathbf{0} \text {,eff }} \ldots \ldots$

A.2.1. The LQ model . . . . . . . . . . . . . . 31

A.2.2. The RCR model . . . . . . . . . . . . . . . 31

$\begin{array}{ll}\text { Acknowledgments } & 32\end{array}$ 


\section{Introduction}

The development of radiation therapy towards more sophisticated treatment techniques such as intensity modulated radiation therapy (IMRT) and light ion therapy, preferably combined with biologically based treatment optimization algorithms and predictive assays, makes it desirable to refine also the mathematical description of tumor and normal tissue dose-response relations. The aim of the present thesis is to explore how improved tumor heterogeneity data, in the form of distributions of radiation resistance, can be used in biologically based radiation therapy optimization, in order to find the best possible radiation modality and treatment technique for the individual patient.

Most tumors are associated with some degree of heterogeneous organization, that may lead to heterogeneities in their response to radiation therapy. Sharp gradients of oxygen and nutrients due to poor tumor vasculature, along with variations in the genetic expression of the tumor cells, give rise to radiation response heterogeneities within the tumor. Patients may also show different sensitivity to radiation therapy. Depending on the radiation modality and dose delivery technique, and the heterogeneity of tissue, the optimal radiation dose delivery should also be heterogeneous. Predictive assays of radiation sensitivity are developing towards the ability to provide geometrical data on vasculature, oxygenation and other important factors for the response of the individual patient. There is thus a clear need to refine and extend the description of the radiation responsiveness of heterogeneous tumors, in order to fully account for individual inter- and intratumoral response heterogeneities in biological treatment optimization.

\subsection{Tumor response heterogeneities in radiation therapy}

\subsubsection{Micro- and macroscopic tumor response heterogeneities}

When heterogeneous tissues are exposed to radiation, interactions take place at a wide range of geometrical scales and energy deposition levels. The radiation response of a heterogeneous tissue with an arbitrary distribution of cellular radiation resistance was quantified in paper III, in terms of the effective response parameters $N_{0, \text { eff }}$ and $D_{0, \text { eff }}$. 
The radiation resistance parameter $D_{0}$ describes the dose required to reduce the clonogen number of a uniform cell population to $1 / e$ or about $37 \%$ of its initial value, based on the exponential cell survival model $S(D)=e^{-D / D_{0}}, c f$. section 3.1. Mathematical expressions for how to obtain $D_{0, \text { eff }}$ for a given heterogeneous cell distribution $N_{D_{0}}$ were given in papers II and III. See also section 3.4.1.

Due to the random nature of atomic and nuclear interactions, it is virtually impossible to deterministically hit individual cells by external beam radiation therapy. The microscopic tissue density variation is not known accurately enough, and neither is the random process of range straggling. So even if we had the ideal ion beams of precisely known energy and negligible multiple scattering, the Bragg peak of each beam having a width of some $50 \mu \mathrm{m}$ (protons) to several mm (carbon), we do not know the integral or mean stopping power along each ray line to better than a few $\mathrm{mm}$ in equivalent range. In addition to this, the range straggling at the tumor depth will be of a similar order of magnitude. The total uncertainty in Bragg peak localization for protons is therefore about $3-4 \mathrm{~mm}$ longitudinally $(1 \sigma)$, whereas multiple Coulomb scattering adds a lateral uncertainty on the order of $5-8 \mathrm{~mm}$ at 15 to $25 \mathrm{~cm}$ depth $(1 \sigma)$. With heavier ions like carbon the range straggling and multiple scattering is reduced by a factor of 3, but now the Bragg peak is instead extended to several mm width longitudinally, making individual cell targeting impossible (Hollmark et al., 2004). Furthermore, the geometric resolution of current tumor imaging systems is limited to around mm-scale at best. There is also considerable organ motion in patients and target setup errors, which have to be accounted for in the target definition. It is thus clear that the response to external beam radiotherapy has to be considered as local mean values in voxels (volume elements) of macroscopic size.

In treatment planning and therapy optimization it is necessary to use the average responsiveness or distributions of radiation sensitivity by using effective cellular radiation response parameters. The voxel size may correspond to the resolution of the imaging system. If the voxels are small (a few mm) and low LET (Linear Energy Transfer) electrons or photons are used, the dose at therapeutic dose levels ( $\sim 60 \mathrm{~Gy}$ ) may be almost constant, within a few percent on the cell nuclear scale. This assumption is valid since the microdosimetric standard deviation on the scale of the cell nucleus $\sigma_{\mu}$ is generally below 1\% (Lindborg and Brahme, 1990; Tilikidis and Brahme, 1994). At higher LET, this microdosimetric uncertainty is greater, possibly creating the need for more elaborate models of cell survival and tumor cure. Also, while using IMRT (Intensity Modulated Radiation Therapy) or high LET, the macroscopic dose gradients can be quite sharp, and then there may be a need for even smaller voxels or, again, more elaborate response models. However, the reduction of voxel size in treatment optimization is limited by computational power, due to the complexity of maximizing the probability of complication-free tumor cure (Löf, 2000). 


\subsubsection{The radiation response of hypoxic cells and tumors}

In order to accurately predict the outcome of radiotherapy, it would be desirable to have the exact distribution of tumor $p_{\mathrm{O}_{2}}$ for every patient, and ultimately before every dose fraction in fractionated radiotherapy. Unfortunately, no convenient way of measuring the oxygenation status of individual tumors on a routinely basis in the clinic has yet been presented. However, there are several techniques that can give at least some information about the $p_{\mathrm{O}_{2}}$ distribution in tissue.

Since tissue oxygenation strongly depends on the efficiency of the vascular network, several methods have been developed that focus on measuring parameters like intercapillary distance (Kolstad, 1968; Awwad et al., 1986), vascular density (Delides et al., 1988; Révész et al., 1989; Hoper and Jahn, 1995; Plasswilm et al., 1999) and the distance from tumor cells to the nearest vessel (Lauk et al., 1989). A way to get a more direct measure of oxygenation is to digitize a microscopic tissue photograph and calculate the corresponding oxygen distribution by solving the diffusion equation for the acquired vascular geometry (Secomb et al., 1993).

These measurements alone will generally not give any information on the amount of oxygen carried in the blood or the functionality of the vessels. Still, they can give quite good indirect estimations of tissue oxygenation, especially when complemented with other measurements. Awwad et al. (1986) showed that the intercapillary distance (ICD) decreased during fractionated radiotherapy of the uterine cervix, and that there is a correlation between high ICD and high probability of local tumor recurrence.

Various agents based on nitroimidazole, such as pimonidazole, EF5 and NITP, can be used to mark hypoxic cells. The nitroimidazole compound misonidazole has been observed to be preferentially metabolized in oxygen deficient cells, and is then covalently bound to the cellular macromolecules for substantial periods of time (Chapman, 1979, 1991; Bussink et al., 1999; Evans and Koch, 2003). Misonidazole can be assessed noninvasively, by ${ }^{18} \mathrm{~F}$-labeling and subsequent viewing with PET (positron emission tomography) (Koh et al., 1991). Several other compounds, labeled with various radioactive isotopes, can be used to mark hypoxic cells for later view with PET or SPECT (single photon emission computed tomography) (Chapman et al., 1998). Such techniques, along with MRI (magnetic resonance imaging), may provide an important step towards individualized radiation therapy (Rasey et al., 1996; Cooper et al., 2000; Chapman et al., 2001; Krishna et al., 2001; Mankoff and Bellon, 2001).

A measurement technique that has been called the "gold standard" among the tumor oxygenation assessment methods is the polarographic $p_{\mathrm{O}_{2}}$ electrode, often called the Eppendorf histograph (Eppendorf GmbH, Hamburg, Germany). This probe consists of a needle (diameter about $300 \mu \mathrm{m}$ ) that has a small oxygen sensor (diameter about 12 $\mu \mathrm{m})$ on the tip. The needle moves forward in small steps, about 0.5 to $1 \mathrm{~mm}$, followed by a small backwards movement to avoid tissue compression. The response time is about one second (Kallinowski et al., 1990; Höckel et al., 1991; Nordsmark et al., 1994). An extensive amount of measurements of the oxygenation status of tumors using the Eppendorf histograph have been reported, some of which have been reviewed 
by Vaupel et al. (1998). A similar technique is the OxyLite probe (Oxford Optronix Ltd., UK), which measures the oxygen concentration by means of fluorescence analysis and fiber optics (Collingridge et al., 1997).

Tumor oxygenation is complex (Vaupel et al., 1989), including concepts such as reoxygenation (van Putten and Kallman, 1968) and chronic/acute hypoxia (Brown, 1979). Even so, the pre-treatment oxygenation status has shown a predictive potential for the outcome of radiation therapy, see for example Evans and Koch (2003) for a review. 


\section{Tissue oxygenation modeling}

\subsection{Overview of existing models of microvascular networks}

If the intercapillary distance in a tumor is large, shells of living cells will form around the vessels. Outside the shells, regions are formed where cells are more or less necrotic. A cylindrical structure is thus formed, where cords of living cells surround the functional blood vessels. This phenomenon was described already by Krogh (1919), assisted by the mathematician Erlang, by assuming a gradient of oxygen pressure outside the vessels. The idea that oxygen is the critical element in forming the cordial structure was supported by histological examination along with calculations of diffusion distances for different nutrients by Thomlinson and Gray (1955). The cylindrical structure of tumor tissue, referred to as the Krogh cylinder model, was analyzed further theoretically by Tannock (1972). Tannock's calculations have in turn been used by several researchers to analyze the oxygen dependence of radiation response, see for example Wouters and Brown (1997), Toma-Daşu et al. (2001, 2002) and paper III.

In healthy normal tissues, vessels are formed in order to ensure that all cells get all the oxygen and nutrition they need to be fully functional. This leads to the development of a microvascular network in the form of a well-ordered tree, with fairly even branch spacing. Tumors, on the other hand, are known to have a rather chaotic structure. Tumor angiogenesis is highly uncontrolled, as well as the proliferation of tumor cells. As a result, the tumor microvasculature is highly irregular, leading to a sub-optimal distribution of oxygen and nutrients (Konerding et al., 1999). Such irregular vascular networks have been successfully modeled by fractal geometry, in the form of invasion percolation. This is a statistical process whereby a network grows into a heterogeneous substrate through the weakest points (Baish et al., 1996; Gazit et al., 1995, 1997). Two measures of network heterogeneity were used: the fractal dimension of the network as a whole, $d_{\text {vasculature }}$, and the minimum path dimension, $d_{\min }$. See also Zamir (2001).

A different approach to modeling tumor microvasculature is to make computer reconstructions of small regions of real tumors (Secomb et al., 1993; Pogue et al., 2001). Secomb and his colleagues observed the vascular geometry in vivo and discretized them in a computer. Calculation of the corresponding oxygen distribution was made by solving the diffusion equation using Green's functions. Oxygen consumption was modeled using Michaelis-Menten kinetics. The conclusion was that the Krogh cylin- 
der model, based on uniform vessel spacing, may seriously underestimate the extent of the hypoxic tissue fraction. Thus, the unstructured nature of tumor microcirculation has a major impact on tumor oxygenation, and therefore also on tumor radiation sensitivity.

Yet another approach is to simulate tumor growth, including growth and radiation resistance parameters for each individual cell, and possibly even angiogenic processes. Such models have been presented by e.g. Kocher and Treuer (1995); Kocher et al. (1997) and Borkenstein et al. (2004), showing that the complex process of tumor growth and response to radiotherapy can be successfully simulated with clinically interesting results.

\subsection{A tissue oxygenation model including vascular heterogeneity}

In paper III, a tissue model was used that takes vascular heterogeneities into account. The model consists of a set of parallel blood vessels, supplying a volume of cells with oxygen. The vessels are placed using a normal (Gaussian) probability distribution in two dimensions, orthogonal to the vessels, where the expectation values for the vessels form a hexagonal grid. Vascular heterogeneity is introduced as the standard deviation of the normal distribution. A small standard deviation leads to a well-ordered vascular structure resembling a healthy tissue, while a large deviation causes a more chaotic vascularity similar to what is seen in tumors (Konerding et al., 1999).

Oxygen diffusion from the vessels is calculated using the solution to the diffusion equation obtained by Tannock (1972) for a single cylindrical vessel, at steady state. The oxygen distributions around the vessels are then superimposed to form the distribution in the whole model tissue. This is a simplified method of calculation, which does not account for the simultaneous oxygen diffusion from all the vessels. In-between nearby vessels, the oxygen concentration can therefore increase beyond the vascular concentration, and the diffusion distance from such non-coupled vessels may be underestimated. Also, oxygen consumption is modeled as independent of the oxygen concentration, which causes negative concentrations beyond a certain distance from the vessels (the "diffusion limit"). The oxygen distribution in the model is therefore cut off at both zero and the vascular level. For a more detailed description, see paper III.

The parallel vascular structure facilitates both the calculation of oxygen diffusion and the visualization of the results. In an orthogonal section through the model tissue, the decrease in oxygen concentration along the vessel length need not be considered. The figure on the front cover shows sections of the tissue model, where the color scale represents the level of oxygen partial pressure $p_{\mathrm{O}_{2}}$ from anoxia (blue) to arterial values (red). Even though the oxygen calculation is simplified, the resulting oxygen distribution histograms are very similar to clinically observed histograms for a wide 
variety of both tumors and healthy tissues, as shown in papers II, III and IV.

A tissue model of this type was used by the present author, Nilsson (1999), for a quadratic vessel grid. Groebe and Vaupel (1988) and Toma-Daşu et al. (2001, 2002) used a similar model, but with homogeneous vessel spacing and a hexagonal grid. A quadratic vessel grid was also used by Grunewald and Sowa (1977), including the decrease in oxygen concentration along the vessels. Vascular heterogeneities were introduced in a different way by Daşu et al. (2003), using distributions (log-normal) of intercapillary distance (ICD). Both the location of the maximum value and the coefficient of variance of the ICD distribution were found to strongly influence the resulting oxygen distribution. Daşu et al. (2003) used a finite element numerical method to calculate the oxygen diffusion, thereby accounting for the coupled vessels. They also accounted for a $p_{\mathrm{O}_{2}}$-dependent oxygen consumption using Michaelis-Menten kinetics, and chronic/acute hypoxia by closing some of the model vessels. A detailed description of the finite element method for oxygen diffusion calculations was given by Pogue et al. (2001). For more thorough discussions on modeling oxygen transport to tissue, see for example Grunewald and Sowa (1977) and Popel (1989). 


\section{The radiation response of heterogeneous tumors}

\subsection{Basic cell survival}

Cells exposed to ionizing radiation are in radiation response modeling generally assumed to either die or survive. The probability that an irradiated cell survives is a complex process that involves concepts such as radiation dose, radiation quality, and cellular radiosensitivity. The radiosensitivity in turn depends on for example the amount of initial damage, the ability to repair DNA damage, the cell cycle phase, the temperature, and also environmental aspects such as the availability of oxygen and nutrition.

In this thesis, the words cell and clonogen are generally synonymous, referring to cells that are clonogenic, i.e. cells that are able to divide infinitely many times. They can thus create or recreate a tumor, and can also be called tumor stem cells. Tumors generally contain many different strains of cells, and not all of them are clonogenic. The tumor cure probability is considered as the probability that all clonogens are eradicated (Munro and Gilbert, 1961; Suit et al., 1965; Porter, 1980), as will be discussed in more detail in section 3.2.

A quite simple approach to modeling the survival probability of irradiated cells was used by Brahme and Ågren (1987). They assumed that the mean number of inactivating hits per cell is the product of $\sigma_{0}$, the inactivation cross section (probability) and $\Phi$, the fluence of ionizing particles. Since an inactivating hit means that the cell is eradicated, the probability that the cell suffers from exactly $\nu$ hits can be described using binomial statistics. When the number of trials (the fluence) is large and the probability of success (inactivation) in each trial is small, while the product of the two is finite, binomial statistics can be approximated with Poisson statistics (see also section 3.2). Brahme and Ågren applied the Poisson approximation, reaching the following expression for the probability of exactly $\nu$ inactivating hits:

$$
p(\nu)=\frac{e^{-\sigma_{0} \Phi}\left(\sigma_{0} \Phi\right)^{\nu}}{\nu !}
$$

The absorbed dose can be expressed as, for low-LET photons and electrons, $D=$ $\Phi \bar{L}_{\Delta} / \rho$, where $\bar{L}_{\Delta} / \rho$ is the restricted mass stopping power. The probability of exactly 
zero hits, i.e. the probability that the cell survives, is then given by

$$
S(D)=p(0)=e^{-\sigma_{0} \Phi}=e^{-D / D_{0}},
$$

where $S(D)$ is the cell survival probability and $D_{0}$ is the dose where $e^{-1} \approx 37 \%$ of the cells survive. The mean number of hits, or the expectation value of $p(\nu)$, is $\sigma_{0} \Phi=D / D_{0}$. Equation (3.2) is also related to the differential equation

$$
\frac{\mathrm{d} N}{\mathrm{~d} D}=-\frac{1}{D_{0}} N
$$

where $\mathrm{d} N / \mathrm{d} D$, the change in number of cells, is proportional to the number of cells $N$. The solution to equation (3.3) is

$$
N(D)=N_{0} e^{-D / D_{0}}=N_{0} S(D),
$$

where $N_{0}$ is the number of initial cells at $D=0$ and $S(D)$ is given by equation (3.2).

In the exponential approximation, damage repair is not considered. Several models have been developed which take repair into account explicitly, including the LQ (linear quadratic), LPL (lethal and potentially lethal), IR (inducible repair) and RCR (repairable-conditionally-repairable) models (Chadwick and Leenhouts, 1973; Douglas and Fowler, 1975; Curtis, 1986; Marples et al., 1997; Lind et al., 2003). At low doses around 1-2 Gy, the repair of sublethal damage gives rise to a shoulder, causing the survival curve to deviate from the purely exponential expression, in a quadratic fashion. Because it describes the shoulder quite well, and is rather simple, the most widely used model of cell survival is the LQ model:

$$
S(D)=e^{-\alpha D-\beta D^{2}}
$$

For high doses, the exponent in the LQ model becomes quadratic, which disagrees with both equation (3.2) and most experimental data (Kellerer and Hug, 1972). A model that describes clonogenic survival both at low, intermediate and high doses, including low-dose hypersensitivity (Marples et al., 2004), is the recent RCR model (Lind et al., 2003):

$$
S(D)=e^{-a D}+b D e^{-c D}
$$

The simple exponential approximation is in general not accurate enough to describe cell survival after single-dose irradiation. However, when repair is low or leading to an exponential survival curve, such as in high-LET beams, it can provide a good fit to the observed survival data. It also provides a good starting point for more elaborate survival modeling. It's greatest use is however in fractionated radiation therapy with a constant dose per fraction. If all repairable lesions are assumed to be repaired from one dose fraction to the next, the survival curve resulting from fractionated dose delivery is accurately described as exponential for homogeneous cell populations. The fractionated cell-survival curve for a constant dose per fraction can thus be described as

$$
S(D)=S(d, n)=S(d)^{n}=e^{-n d / D_{0}},
$$


where $d$ is the dose per fraction, $S(d)$ is the surviving fraction at $d, n$ is the number of fractions and $D=n d$ is the total dose, $c f$. paper IV. For heterogeneous populations, the fractionated survival can still be described based on equation (3.4), as discussed in papers I, II, III and IV, along with sections 3.2 and 3.3.

\subsection{Heterogeneous radiation response based on binomial and Poisson statistics}

In traditional radiation biology, cell survival is often regarded as a binomial process were the cells are either alive or dead, referring to their ability or inability to form colonies. Binomial statistics is generally approximated with Poisson statistics, since the number of cells is large while the probability of cell survival is low. However, when cells are divided into groups of different sensitivities, compartments may occur with only a small number of cells, rendering the Poisson approximation less accurate. In papers II, III and IV the Poisson approximation was used exclusively. Here we will generalize the dose-response calculations further, including a full multi-compartment binomial analysis.

The number of surviving cells $n$ of $N_{0}$ independent clonogenic cells, with each cell having the dose-dependent survival probability $S(D)$, has a binomial distribution with the probability function

$$
P_{\mathrm{b}}=\left(\begin{array}{c}
N_{0} \\
n
\end{array}\right) S^{n}(1-S)^{N_{0}-n}
$$

For the tumor control probability, the interesting case is when no cells survive, i.e. when $n=0$, yielding

$$
\left.P_{\mathrm{b}}\right|_{n=0}=(1-S)^{N_{0}}=e^{N_{0} \ln (1-S)} .
$$

When $S$ is small and $N_{0}$ large, while $N_{0} S$ is finite, the binomial distribution turns into the Poisson distribution (see for example Brahme and Agren (1987); Johnson et al. (1993)):

$$
P_{\mathrm{P}}=\frac{\left(N_{0} S\right)^{n} e^{-N_{0} S}}{n !}
$$

which when no cells survive $(n=0)$ becomes

$$
\left.P_{\mathrm{P}}\right|_{n=0}=e^{-N_{0} S}
$$

A unique feature of the Poisson distribution is that the expectation value $\mathrm{E} P$ is equal to the variance $\mathrm{V} P$, which in our case means that $\mathrm{E} P=\mathrm{V} P=N_{0} S$. The expectation value is the mean number of surviving clonogens

$$
N(D)=N_{0} S(D)
$$

at the dose $D$, which was derived for exponential survival in equation (3.3). 
For heterogeneous radiation resistance with $k$ independent clonogen compartments, the total dose-response relation becomes $P=\prod_{i=1}^{k} P_{i}$, where $P_{i}$ is the tumor cure probability for compartment $i$. Each compartment also has a probability of clonogen survival $S_{i}$ and an initial number of clonogens $N_{0, i}$. Using binomial statistics, we have

$$
P_{\mathrm{b}}=\prod_{i=1}^{k}\left(1-S_{i}\right)^{N_{0, i}}=\exp \left[\sum_{i=1}^{k} N_{0, i} \ln \left(1-S_{i}\right)\right],
$$

and for Poisson statistics,

$$
P_{\mathrm{P}}=\prod_{i=1}^{k} e^{-N_{0, i} S_{i}}=\exp \left[-\sum_{i=1}^{k} N_{0, i} S_{i}\right] .
$$

At the limit to a continuous distribution, the clonogen distribution is described by $N_{D_{0}}$ (cf. papers II and III), and the sums are transformed into integrals according to

$$
P_{\mathrm{b}}=\exp \left[\int N_{D_{0}} \ln \left\{1-S\left(D, D_{0}\right)\right\} \mathrm{d} D_{0}\right]
$$

for binomial statistics and

$$
P_{\mathrm{P}}=\exp \left[-\int N_{D_{0}} S\left(D, D_{0}\right) \mathrm{d} D_{0}\right]
$$

for the Poisson approximation. In both cases, the total number of surviving clonogens $N(D)$ is the weighted sum of the contributions from each compartment, although with different weighting functions $(\ln (1-S)$ or $-S)$. The latter expression was used to describe the radiation response of heterogenous tumors in paper II, and with $S\left(D, D_{0}\right)$ defined by equation (3.2) in papers III and IV.

Another way of deriving the probability of tumor cure for heterogeneous cell populations is to assume that $D_{0}$ is a discrete stochastic variable with the probability function $p_{D_{0}}\left(D_{i}\right)$, where $D_{i}=D_{0}(i)$, see also section 3.7. The expectation (mean) value of $S\left(D, D_{0}\right)$ is then

$$
\mathrm{E} S\left(D, D_{0}\right)=\sum_{i=1}^{k} S\left(D_{i}\right) p_{D_{0}}\left(D_{i}\right)
$$

By assuming that the survival probability for the whole tumor is equal to the expectation value $\mathrm{E} S\left(D, D_{0}\right)$, while using the Poisson approximation from equation (3.11), we get the probability of tumor control as

$$
P_{\mathrm{P}}\left(D, D_{0}\right)=e^{-N_{0} \mathrm{E} S\left(D, D_{0}\right)}=\exp \left[-N_{0} \sum_{i=1}^{k} S\left(D_{i}\right) p_{D_{0}}\left(D_{i}\right)\right] .
$$


Since a probability function such as $p_{D_{0}}\left(D_{i}\right)$ is normalized by definition so that $\sum_{i=1}^{k} p_{D_{0}}\left(D_{i}\right) \equiv 1$, and $\sum_{i=1}^{k} N_{0, i}=N_{0}$, where $N_{0, i}$ is the number of initial clonogens in compartment $i$, we can define $p_{D_{0}}\left(D_{i}\right)$ as

$$
p_{D_{0}}\left(D_{i}\right)=\frac{N_{0, i}}{\sum_{i=1}^{k} N_{0, i}}=\frac{N_{0, i}}{N_{0}} .
$$

Using this definition, equation (3.18) becomes $P\left(D, D_{0}\right)=\exp \left[-\sum_{i=1}^{k} N_{0, i} S\left(D_{i}\right)\right]$, which is equivalent to equation (3.14). This approach was used by Lind and Brahme (1997) for continuous stochastic variables. For the continuous case, the probability function is $p_{D_{0}}=N_{D_{0}} / N_{0}$, and we can see that equation (3.18) then transforms into equation (3.16).
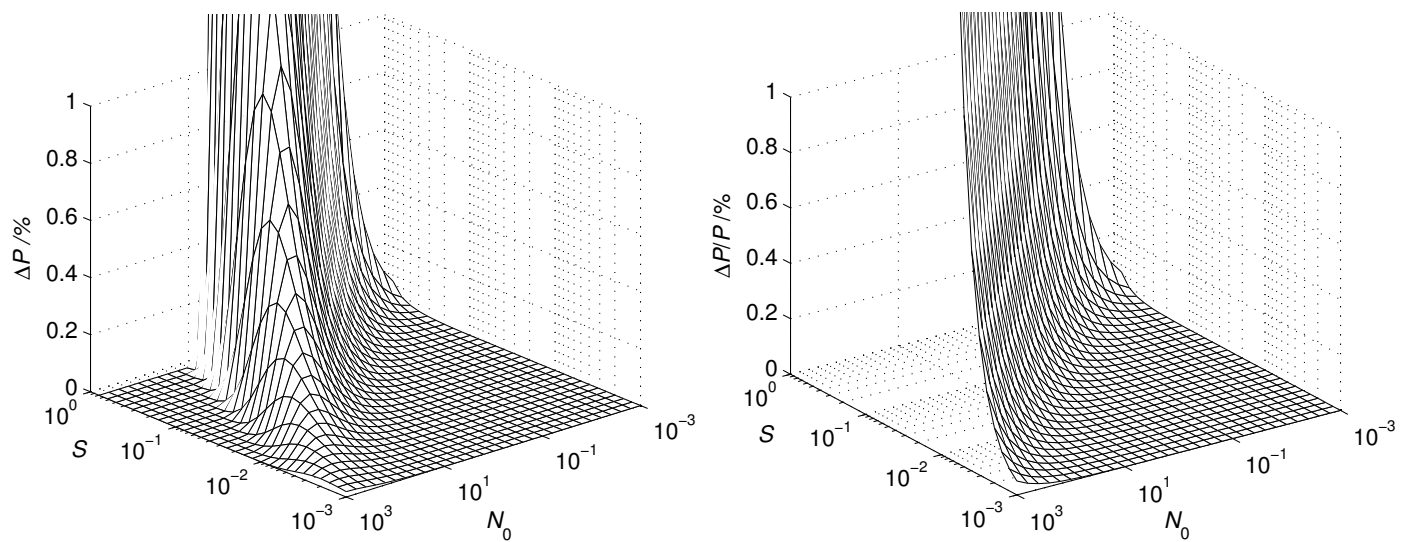

Figure 3.1.: The absolute (left) and relative (right) difference between the probability of tumor cure modeled with binomial and Poisson statistics.

The deviation of the Poisson approximation from binomial statistics can be analyzed by a Taylor expansion of the relative difference between equations (3.11) and (3.9), around a small $S$ and a small $N_{0}$. This results in

$$
\begin{aligned}
\frac{\Delta P}{P}=\frac{e^{-N_{0} S}-(1-S)^{N_{0}}}{(1-S)^{N_{0}}=\frac{N_{0}}{2} S^{2}} & +\frac{N_{0}}{3} S^{3}+\frac{2 N_{0}+N_{0}^{2}}{8} S^{4}+ \\
& +\frac{6 N_{0}+5 N_{0}^{2}}{30} S^{5}+O\left(N_{0}^{6}\right) O\left(S^{2}\right),
\end{aligned}
$$

which shows that the Poisson approximation is quite good when $N_{0} S^{2} / 2$ is small. A similar result is achieved by expanding the absolute difference. We can see from equation (3.11) that at $D_{37}$, where $P=e^{-1} \approx 37 \%$, only one clonogen remains (on average) while using Poisson statistics, since $N_{0} S\left(D_{37}\right)=1$. To reach a reasonable probability of tumor cure, higher doses than $D_{37}$ need to be administered. We can also see from equation (3.11) that $S\left(D_{37}\right)=1 / N_{0}$, so for the dose range of interest 
for tumor control, we have the condition that $S<1 / N_{0}$. For e.g. $P=90 \%$ we have that $S \approx 0.1 / N_{0}$ and thus $N_{0} S^{2} \approx 0.01 / N_{0}$, which would certainly qualify as small enough to show that the Poisson approximation is very accurate, for any reasonable value of $N_{0}$.

It follows that at therapeutic doses, the Poisson approximation is accurate even at a very small number of clonogens. In fact, inserting $S=0.1 / N_{0}$ (corresponding to $P \approx 90 \%$ ) and $N_{0}=1$ (a single clonogen) into equations (3.9) and (3.11) gives $\left(P_{\mathrm{P}}-P_{\mathrm{b}}\right) / P_{\mathrm{b}} \approx 0.54 \%$, i.e. a relative error of around half a percent. In other words, the Poisson approximation to binomial tumor cure statistics at curative doses is very good even for extremely low clonogen numbers. Figure 3.1 shows the absolute and relative error in $P(D)$ from using the Poisson approximation, as functions of $S$ and $N_{0}$.

It is thus possible to use Poisson statistics for modeling the control probability of tumors or tumor compartments with a very small number of clonogens, when a high dose is ensured in the whole tumor volume. However, there are cases when the Poisson approximation may show substantial deviations from the binomial distribution. If cold spots of low dose occur in the irradiated tumor, $S$ may be comparatively high in those locations, requiring the use of binomial statistics. The cold spots may substantially deteriorate the Poisson approximation, even if they cover only a very small number of cells. Also, radiation damage to healthy tissues may be induced at much lower doses, and thus higher values of $S$, than what is needed for tumor cure. When modeling the radiation response of healthy tissues and organs as well as strongly heterogeneous dose delivery, binomial statistics is definitely the preferred method. During the course of fractionated radiation therapy, the compartments may not remain independent, in which case neither binomial nor Poisson statistics may be sufficient.

Describing all relevant dynamic cellular processes in detail would be very difficult with our present knowledge of the underlying biological mechanisms. Therefore, effective response parameters based on clinically observed dose-response data have generally been used, $c f$. Brahme (1984); Brahme and Ågren (1987); Källman et al. (1992) and papers I, II, III and IV. Since the clinical data already account for all the underlying biological processes, the derived effective response parameters will reflect the clinically relevant response phenomena. When the effective clonogen numbers are determined from the cell kill produced by the delivered doses, all biological processes, such as the five Rs of radiotherapy (Withers, 1975; Steel et al., 1989), are accounted for in the final treatment plan. More recently, inter-fraction tumor dynamics such as cell proliferation have been separately considered (Tucker and Taylor, 1996; Deasy, 1996; Tucker et al., 1990).

\subsection{Generalized cell survival}

For a uniform dose a general cell survival model can be expressed in the form $S(\vec{\chi}, D)$, where the dose is a scalar quantity and $\vec{\chi}$ is a vector of model parameters describing 
the radiation sensitivity. Examples of such parameters are $\vec{\chi}=D_{0}$ for the simple exponential model (equation (3.2)), $\vec{\chi}=(\alpha, \beta)$ for the LQ model, and $\vec{\chi}=(a, b, c)$ for the more general RCR model, see section 3.1. This may apply to a single voxel where the dose can be assumed to be uniform, and where the survival parameters vary due to variations in for example cellular oxygenation. More generally, the dose delivery may be expressed as a dose distribution vector $\vec{D}$ describing the spatial dose distribution over all voxels of interest in a tumor or normal tissue. The dose vector may also be applied at a more macroscopic level, where each dose vector element corresponds to a voxel.

Heterogeneous sensitivity distributions may exist due to variations in genetic predisposition, single nucleotide polymorphism, hypoxia, dose distribution heterogeneity, radiation quality distributions and other factors of significance for the micro- and macroscopic radiation response. The general radiation response can be expressed for a continuous clonogen distribution function $N_{D_{0}}$ which is differential in $D_{0}$, and thus describes the number of clonogens with different $D_{0}$ values, $c f$. papers II and III. $N_{D_{0}}$ can account for any or all of the many reasons for heterogeneous sensitivity described above. The total number of initial clonogens is then given by $N_{0}=\int N_{D_{0}} \mathrm{~d} D_{0}$.

When using more elaborate survival models than purely exponential, other parameters than $D_{0}$ may be of interest to describe the radiation sensitivity or resistance. All such parameter sets $\vec{\chi}$ may depend in different ways on the DNA repair capacity, the availability of oxygen and nutrition, the LET and other factors of importance for the radiation responsiveness. This dependency may be expressed as $\vec{\chi}(\vec{\Upsilon})$, where $\vec{\Upsilon}$ is a vector consisting of elements such as $p_{\mathrm{O}_{2}}$ and LET. In paper III, the simple case of $\vec{\chi}=D_{0}$ and $\vec{\Upsilon}=p_{\mathrm{O}_{2}}$ was investigated. In paper IV, this is extended to $\vec{\chi}=(\alpha, \beta)$ and $\vec{\Upsilon}=\left(p_{\mathrm{O}_{2}}\right.$, LET $)$. If the dependency is known, it is possible to use the associated clonogen sensitivity distribution function $N_{\chi(\vec{\Upsilon})}$ which is differential in $\chi$, one of the elements of $\vec{\chi}$. The corresponding generalized survival function with a uniform dose delivery can then be expressed as $S(\chi(\vec{\Upsilon}), D)$, and the initial number of clonogens becomes $N_{0}=\int N_{\chi} \mathrm{d} \chi$. The number of surviving clonogens $N$ at the dose level $D$ can then be expressed according to

$$
N(D)=\int N_{\chi} S(\chi, D) \mathrm{d} \chi
$$

The mean or effective cell survival can be defined as

$$
\bar{S}(D)=\frac{N(D)}{N_{0}}=\frac{\int N_{\chi} S(\chi, D) \mathrm{d} \chi}{\int N_{\chi} \mathrm{d} \chi} .
$$

In the discrete case, the clonogen number and survival can be described as vectors, with each element corresponding to one compartment of clonogens. Each compartment consists of all clonogens within a certain interval of the parameter $\chi$. The mean number of surviving cells can then be described as the scalar product of the vectors:

$$
N(D)=\vec{N}_{\chi} \cdot \vec{S}(\chi, D)
$$


Using the vector formalism and the scalar product can be convenient in mathematical computer software that is based on matrix algebra.

\subsection{The effective radiation response}

For an arbitrary micro- or macroscopic distribution of radiation sensitivity or absorbed dose it may be desirable to calculate effective radiation response parameters, as discussed by Brahme (1984) and in papers II, III and IV. This can be done in terms of $N_{0, \text { eff }}$ together with an effective parameter vector $\vec{\chi}_{\text {eff }}$ for a specific cell survival model, including parameters such as $D_{0, \text { eff }},\left\{\alpha_{\text {eff }}, \beta_{\text {eff }}\right\}$ of the LQ model or $\left\{a_{\text {eff }}, b_{\text {eff }}, c_{\text {eff }}\right\}$ of the RCR model, $c f$. section 3.3. This vector may be accompanied by an effective absorbed dose, $D_{\text {eff }}$.

The number of surviving cells can be approximated by the effective survival function

$$
N(D)=\int N_{\chi} S(\chi, D) \mathrm{d} \chi \approx N_{0, \mathrm{eff}} S\left(\vec{\chi}_{\mathrm{eff}}, D\right)
$$

Differentiation of this relation yields

$$
\frac{\partial N(D)}{\partial D}=\int N_{\chi} S^{\prime}(\chi, D) \mathrm{d} \chi \approx N_{0, \mathrm{eff}} S^{\prime}\left(\vec{\chi}_{\mathrm{eff}}, D\right)
$$

where $S^{\prime}(\chi, D)=\partial S(\chi, D) / \partial D$. If the vector $\chi$ has $n$ components, $n+1$ equations are needed to determine all components, i.e. the derivative of $N(D)$ has to be determined to the $n^{\text {th }}$ order.

At a given dose level of interest $D$ the relations (3.24) and (3.25) are exact. When more than one effective parameter besides $N_{0 \text {,eff }}$ need to be determined, these relations and the associated higher order partial derivatives may make it possible to analytically or numerically deduce effective values for arbitrary distributions in radiation sensitivity. At $D_{37}$, the dose where the probability of tumor cure is $e^{-1} \approx 37 \%$, the mean number of surviving cells $N(D)$ is one, $c f$. equation (3.24) and Porter (1980). Choosing $D_{37}$ as the level where the effective response parameters are exact will therefore lead to simpler analytical expressions.

\subsubsection{Effective exponential response}

For constant-dose fractionated radiation therapy of a homogeneous cell population, the cell survival curve becomes purely exponential, as was described in section 3.1 and paper IV. In this case, the parameter $D_{0, \text { eff }}$ replaces $\vec{\chi}_{\text {eff }}$ in equations (3.24) and (3.25), and the resulting effective radiation response is, as expressed in paper III,

$$
N(D)=N_{0, \text { eff }} e^{-D / D_{0, \text { eff }}}
$$


which describes an exponential tangent to the true $N(D)$. The tangent is equal to $N(D)$ at a certain dose level $D$, where the effective parameters are defined. The effective parameters for basic exponential survival (equation (3.4)) of one compartment of radiation resistance were derived in paper III at an arbitrary dose level $D$ as

$$
D_{0, \mathrm{eff}}=\frac{N(D)}{-N^{\prime}(D)}=\frac{\int N_{D_{0}} e^{-D / D_{0}} \mathrm{~d} D_{0}}{\int \frac{N_{D_{0}}}{D_{0}} e^{-D / D_{0}} \mathrm{~d} D_{0}}
$$

and

$$
N_{0, \text { eff }}=N(D) e^{D / D_{0, \text { eff }}}=\left[\int N_{D_{0}} e^{-D / D_{0}} \mathrm{~d} D_{0}\right] \exp \left(\frac{\int N_{D_{0}} \frac{D}{D_{0}} e^{-D / D_{0}} \mathrm{~d} D_{0}}{\int N_{D_{0}} e^{-D / D_{0}} \mathrm{~d} D_{0}}\right) .
$$

Here, $N_{D_{0}}$ is the radiation resistance distribution, or the number of clonogens differential in $D_{0}$ so that $\int N_{D_{0}} \mathrm{~d} D_{0}=N_{0}$. At $D_{37}$, the effective values can be expressed in a simpler way, since $N\left(D_{37}\right) \equiv 1$ :

$$
D_{0, \mathrm{eff}, D_{37}}=\frac{1}{\int \frac{N_{D_{0}}}{D_{0}} e^{-D_{37} / D_{0}} \mathrm{~d} D_{0}}
$$

and

$$
N_{0, \text { eff }, D_{37}}=e^{D_{37} / D_{0, \text { eff }, D_{37}}}=e^{D_{37} \int N_{D_{0}} D_{0}^{-1} e^{-D_{37} / D_{0}} \mathrm{~d} D_{0}} .
$$

At $D=0$, where $N(0)=\int N_{D_{0}} \mathrm{~d} D_{0}=N_{0}$, the initial effective values are

$$
D_{0, \mathrm{eff}, 0}=\frac{N_{0}}{-N^{\prime}(0)}=\frac{N_{0}}{\int \frac{N_{D_{0}}}{D_{0}} \mathrm{~d} D_{0}}
$$

and

$$
N_{0, \mathrm{eff}, 0}=N_{0}
$$

The effective values for two compartments are described in section 3.6.2.

With the simple one-compartment model it is also possible to derive an effective $D_{37}$ value using equations (3.27) and (3.28), since $N_{0, \text { eff }} e^{-D_{37, \text { eff }} / D_{0, \text { eff }}} \equiv 1$, and thus, as was also discussed in paper III,

$$
D_{37, \text { eff }}=D_{0, \text { eff }} \ln N_{0, \text { eff }}=D+\frac{\int N_{D_{0}} e^{-D / D_{0}} \mathrm{~d} D_{0}}{\int \frac{N_{D_{0}}}{D_{0}} e^{-D / D_{0}} \mathrm{~d} D_{0}} \ln \int N_{D_{0}} e^{-D / D_{0}} \mathrm{~d} D_{0} .
$$

Here it must be remembered that $D$ is in this case the dose where the effective values are exact. Obviously, if the effective values are defined at $D_{37}$, then $D_{37 \text {,eff }}=D_{37}$. If

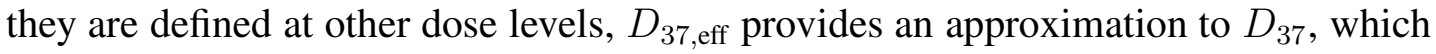
may be useful since $D_{37}$ can not generally be extracted analytically in a closed form from the relation $\int N_{D_{0}} e^{-D_{37} / D_{0}} \mathrm{~d} D_{0} \equiv 1$. In fact, equation (3.33) is very similar to the first iteration of Newton's method as an approximation to $D_{37}$. 
In paper III, it was shown that by stepping up and down the dose-response curve $P(D)$ from $D_{37}$ in steps of $D_{0, \text { eff }}$, the interesting parts of the curve can be reached in a simple fashion. For $d=\left(D-D_{37}\right) / D_{0 \text {,eff }}$ the dose-response becomes $P(d)=$ $\exp (-\exp (-d))$. If $d=-1,0,1,2,3$ then $P(d) \approx 0.066,0.37,0.69,0.87$ and 0.95 . In fact, Porter (1980) pointed out that while using Poisson statistics, the dose difference between the tumor control probabilities 0.37 and 0.69 is approximately $D_{0}$, which in the present terms would equal $D_{0, \text { eff }}$. A rough estimate of $D_{0, \text { eff }}$ is thus achieved by taking the dose difference between one third and two thirds of the dose-response curve.

\subsection{The generalized dose-response gradient}

The normalized dose-response gradient, $\gamma$, was introduced 20 years ago by Anders Brahme (1984), and is widely used to characterize the radiation dose-response relation for both tumors and normal tissues. Brahme showed that, while describing the doseresponse relation with Poisson statistics, $c f$. section 3.2, the $\gamma$ value at $37 \%$ tumor control is proportional to the logarithm of the effective clonogen number $N_{0}$, or more specifically, $\tilde{\gamma}=e^{-1} \ln N_{0}$. This was the first time that effective response parameters were discussed in radiation response modeling. In papers III and IV, it was shown that $\tilde{\gamma}=e^{-1} \ln N_{0, \text { eff }}$, if $N_{0, \text { eff }}$ is defined at $D_{37}$.

Due to its dependence on the effective clonogen number, $\gamma$ should theoretically increase with tumor volume. This increase with tumor size was seen in clinical larynx cancer data by Ågren-Cronqvist et al. (1995). Using an experimental mouse model, Khalil et al. (1997) showed a slow increase in $\gamma$ with tumor volume under ambient conditions. Under hypoxic conditions (clamped tumors), the increase with volume was more expressed, causing higher $\gamma$ values. This result agrees well with the findings presented in figure 4 of paper IV. If a tumor becomes more hypoxic, the effective number of resistant clonogens may increase, causing a higher $\gamma$ value. However, the increase in $\gamma$ due to an increased number of clonogens may be counteracted by an increased heterogeneity, both within tumors and between different patients. This could result in a lower effective clonogen number, and thus a lower $\gamma$, than what would be expected from a simple proportionality between the number of clonogens and the tumor volume (Bentzen and Thames, 1996).

In paper I the normalized dose-response gradient $\gamma$ was generalized to account for non-uniform dose distributions (and possibly also distributions in radiation sensitivity) by the definition

$$
\gamma(\vec{D})=\vec{D} \cdot \nabla_{D} P(\vec{D}) .
$$

which is the scalar product of the dose vector and the gradient of the dose-response relation. This emphasizes the fact that $\gamma$ is a function of the dose distribution and becomes a useful tool for the evaluation and interpretation of radiobiologically optimized treatment plans. In a given treatment plan, the generalized $\gamma$ for an organ or tumor might deviate considerably from the fixed $\tilde{\gamma}$ or $\hat{\gamma}$. Still, the important property 
that

$$
\Delta P \approx \frac{\Delta D}{D} \gamma
$$

holds (Brahme, 1984), which means that the generalized $\gamma$ can be used to estimate the expected change in local response as a function of dose. If $e . g$. an organ at risk has a very high generalized $\gamma$, the probability of complications in that specific organ will decrease more than in an organ with a less high $\gamma$ if the treatment plan is renormalized to a lower dose. In a sense, the generalized $\gamma$ has the same property as a Lagrange multiplier, describing the sensitivity of an optimal treatment plan on the dose level, but with a more simple clinical interpretation.

\subsection{The resistant-sensitive ( $r-s)$ cell survival model}

The r-s model for two clonogen compartments with different effective radiation resistances was defined in paper IV as

$$
N(D)=N_{\mathrm{r}} e^{-D / D_{\mathrm{r}}}+N_{\mathrm{s}} e^{-D / D_{\mathrm{s}}} .
$$

As discussed in paper IV, a two-compartment model of cell survival has been used extensively in radiobiological modeling studies, especially while evaluating the influence of hypoxic fractions. However, paper IV provides a more in-depth study of the analytical properties of this simplified way of analyzing heterogeneous clonogen populations.

The transition dose $D_{\mathrm{t}}$, where the resistant clonogens start to dominate the radiation response, was defined in paper IV. This dose is at the maximum change of steepness of the logarithmic fractionated survival curve. In the case of the r-s model, $D_{\mathrm{t}}$ is also the dose where there is an equal number of surviving clonogens in both compartments, so that $N_{\mathrm{s}} e^{-D_{\mathrm{t}} / D_{\mathrm{s}}} \equiv N_{\mathrm{r}} e^{-D_{\mathrm{t}} / D_{\mathrm{r}}}$, as was derived in paper IV. It was shown that

$$
D_{\mathrm{t}}=\frac{\ln N_{\mathrm{s}}-\ln N_{\mathrm{r}}}{D_{\mathrm{s}}^{-1}-D_{\mathrm{r}}^{-1}}=\frac{D_{\mathrm{r}} D_{\mathrm{s}}}{D_{\mathrm{r}}-D_{\mathrm{s}}} \ln \frac{N_{\mathrm{s}}}{N_{\mathrm{r}}}=-D_{\delta} \ln \nu,
$$

where $D_{\delta}$ is the difference in radiation sensitivity between the two compartments, and $\nu$ is the resistant to sensitive cell ratio, see paper IV. The slope of the survival curve for a two-compartment model will decrease as the resistant cells start to dominate the response. As already mentioned, the maximum decrease occurs at $D_{\mathrm{t}}$. As shown in figure 3.2, the slope of the corresponding tumor cure probability will also decrease at $D_{\mathrm{t}}$. However, the decrease in slope is not as marked as in the survival curve.

\subsubsection{Reduction of the r-s model into one component}

The effective parameters corresponding to exponential survival of heterogeneous radiation resistance were derived in paper III, $c f$. also section 3.4.1 and paper II. For the 
r-s model, defined by equation (3.36), the general effective values reduce to

$$
D_{0, \mathrm{eff}}=\frac{N_{\mathrm{r}} e^{-D / D_{\mathrm{r}}}+N_{\mathrm{s}} e^{-D / D_{\mathrm{s}}}}{\frac{N_{\mathrm{r}}}{D_{\mathrm{r}}} e^{-D / D_{\mathrm{r}}}+\frac{N_{\mathrm{s}}}{D_{\mathrm{s}}} e^{-D / D_{\mathrm{s}}}}
$$

and

$$
N_{0, \text { eff }}=\left(N_{\mathrm{r}} e^{-D / D_{\mathrm{r}}}+N_{\mathrm{s}} e^{-D / D_{\mathrm{s}}}\right) \exp \left[\frac{D\left(\frac{N_{\mathrm{r}}}{D_{\mathrm{r}}} e^{-D / D_{\mathrm{r}}}+\frac{N_{\mathrm{s}}}{D_{\mathrm{s}}} e^{-D / D_{\mathrm{s}}}\right)}{N_{\mathrm{r}} e^{-D / D_{\mathrm{r}}}+N_{\mathrm{s}} e^{-D / D_{\mathrm{s}}}}\right] .
$$

If the effective values are defined at $D_{37}$, the effective parameters can be expressed in a simpler way, since by definition $N_{\mathrm{r}} e^{-D_{37} / D_{\mathrm{r}}}+N_{\mathrm{s}} e^{-D / D_{\mathrm{s}}} \equiv 1$. If the effective parameters are defined at $D_{\mathrm{t}}$, defined by equation (3.37), the effective $D_{37}, c f$. equation (3.33), becomes:

$$
D_{37, \text { eff, } D_{\mathrm{t}}}=D_{\mathrm{t}}+D_{\mathrm{h}} \ln N_{\mathrm{t}}
$$

where $D_{\mathrm{h}}$ is the harmonic mean radiation resistance, $c f$. paper IV and equation (3.43).

\subsubsection{The effective radiation response when the resistant or sensitive clonogens dominate}

The heterogeneous clonogen survival as described by the r-s model was divided into three dose regions, and three corresponding tumor types, in paper IV. The survival curve for each tumor type can, as we will see, be approximated by an exponential tangent, defined by the effective radiation resistance $D_{0, \text { eff }}$ and effective initial clonogen number $N_{0, \text { eff }}$ from papers II and III.

The exponential tangent to $N(D)$ when the resistant clonogens dominate, and $N_{\mathrm{t}}>$ 2 , can be given by the effective parameters evaluated at $D_{37, \mathrm{r}}=D_{\mathrm{r}} \ln N_{\mathrm{r}}$, according to equations (3.27) and (3.28):

$$
\begin{gathered}
D_{0, \text { eff }}\left(D_{37, \mathrm{r}}\right)=\frac{1+N_{\mathrm{s}} N_{\mathrm{r}}^{-D_{\mathrm{r}} / D_{\mathrm{s}}}}{\frac{1}{D_{\mathrm{r}}}+\frac{N_{\mathrm{s}}}{D_{\mathrm{s}}} N_{\mathrm{r}}^{-D_{\mathrm{r}} / D_{\mathrm{s}}}} \\
N_{0, \text { eff }}\left(D_{37, \mathrm{r}}\right)=\left(1+N_{\mathrm{s}} N_{\mathrm{r}}^{-D_{\mathrm{r}} / D_{\mathrm{s}}}\right) N_{\mathrm{s}}^{\frac{1+D_{\mathrm{r}} D_{\mathrm{s}}^{-1} N_{\mathrm{s}} N_{\mathrm{r}}^{-D_{\mathrm{r}} / D_{\mathrm{s}}}}{1+N_{\mathrm{s}} N_{\mathrm{r}}^{-} D_{\mathrm{r}} / D_{\mathrm{s}}}}
\end{gathered}
$$

In the intermediate region, where $1 \leq N_{\mathrm{t}} \leq 2$ and both the sensitive and resistant clonogens strongly influence the radiation response, the exponential tangent to $N(D)$ can be found by evaluating the effective values at $D_{\mathrm{t}}$ :

$$
\begin{gathered}
D_{0, \text { eff }}\left(D_{\mathrm{t}}\right)=\frac{2}{\frac{1}{D_{\mathrm{r}}}+\frac{1}{D_{\mathrm{s}}}}=\frac{2 D_{\mathrm{r}} D_{\mathrm{s}}}{D_{\mathrm{r}}+D_{\mathrm{s}}}=D_{\mathrm{h}} \\
N_{0, \text { eff }}\left(D_{\mathrm{t}}\right)=2 \sqrt{N_{\mathrm{r}} N_{\mathrm{s}}}
\end{gathered}
$$


Notice that $D_{0, \text { eff }}\left(D_{\mathrm{t}}\right)$ is equal to the harmonic mean value of $D_{\mathrm{s}}$ and $D_{\mathrm{r}}$, i.e. the harmonic mean radiation resistance $D_{\mathrm{h}}$ (see also equation (3.43), section 3.7 and paper IV), while $N_{0, \text { eff }}\left(D_{\mathrm{t}}\right)$ is twice the geometric mean value of $N_{\mathrm{s}}$ and $N_{\mathrm{r}}$.

The exponential tangent to $N(D)$ when the sensitive clonogens dominate, and $N_{\mathrm{t}}<$ 1 , is well described by the effective parameters evaluated at $D_{37, \mathrm{~s}}$ :

$$
\begin{gathered}
D_{0, \text { eff }}\left(D_{37, \mathrm{~s}}\right)=\frac{1+N_{\mathrm{r}} N_{\mathrm{s}}^{-D_{\mathrm{s}} / D_{\mathrm{r}}}}{\frac{1}{D_{\mathrm{s}}}+\frac{N_{\mathrm{r}}}{D_{\mathrm{r}}} N_{\mathrm{s}}^{-D_{\mathrm{s}} / D_{\mathrm{r}}}} \\
N_{0, \text { eff }}\left(D_{37, \mathrm{~s}}\right)=\left(1+N_{\mathrm{r}} N_{\mathrm{s}}^{-D_{\mathrm{s}} / D_{\mathrm{r}}}\right) N_{\mathrm{s}}^{\frac{1+D_{\mathrm{s}} D_{\mathrm{r}}^{-1} N_{\mathrm{r}} N_{\mathrm{s}}^{-D_{\mathrm{s}} / D_{\mathrm{r}}}}{1+N_{\mathrm{r}} N_{\mathrm{s}}^{-D_{\mathrm{s}} / D_{\mathrm{r}}}}}
\end{gathered}
$$

Figure 3.2 shows the survival curves and dose-response relations corresponding to the three tumor types defined in paper IV. The effective value approximations given above for each type are shown with dashed lines. In all three cases in the figure, $D_{37}$ is quite close to $D_{\mathrm{t}}$, so that $N_{\mathrm{c}} \approx 1$. Therefore, the upwards bending of the survival curve occurs at the slope of the dose-response relation, and the effective value approximation overestimates the tumor cure probability. This is especially true in the middle panel of figure 3.2, where $D_{37}$ is closest to $D_{\mathrm{t}}$.

\subsubsection{The resistant fraction}

In paper IV, the $\tilde{\gamma}$ value was plotted as a function of the resistant to sensitive clonogen ratio $\nu$ for a fixed number of clonogens $N_{0}$ and a fixed fractional dose $d$.

The resistant fraction $\eta$ of the total number of clonogens can be defined according to

$$
\eta \equiv \frac{N_{\mathrm{r}}}{N_{\mathrm{r}}+N_{\mathrm{s}}}=\frac{\nu}{\nu+1},
$$

where $\nu=N_{\mathrm{r}} / N_{\mathrm{s}}$ is the resistant to sensitive cell number ratio, $c f$. paper IV. If $\nu \ll 1$, which is commonly the case, then $\nu \approx \eta$. In paper IV the r-s model was expressed using $\nu$. Using $\eta$, the $\mathrm{r}-\mathrm{s}$ model instead becomes

$$
N(D)=N_{0}\left[\eta e^{-D / D_{\mathrm{r}}}+(1-\eta) e^{-D / D_{\mathrm{s}}}\right],
$$

where $N_{0}=N_{\mathrm{r}}+N_{\mathrm{s}}$.

\subsection{The frequency function of $D_{0}$ and the mean and effective values of $D_{0}$ and $N_{0}$}

If we treat $D_{0}$ as a stochastic variable, see also equations (3.17) to (3.19), the associated frequency function at dose $D$ is given by

$$
\varphi_{D_{0}}(D)=\frac{N_{D_{0}} S\left(D, D_{0}\right)}{\int N_{D_{0}} S\left(D, D_{0}\right) \mathrm{d} D_{0}} .
$$


The frequency function $\varphi_{D_{0}}$ can be seen as the clonogen survival function $S_{D_{0}}$, differential in $D_{0}$. Using exponential survival, see equation (3.2), $\varphi_{D_{0}}$ at dose level $D$ becomes

$$
\varphi_{D_{0}}(D)=\frac{N_{D_{0}} e^{-D / D_{0}}}{\int N_{D_{0}} e^{-D / D_{0}} \mathrm{~d} D_{0}}=\frac{N_{D_{0}} e^{-D / D_{0}}}{N(D)} .
$$

The expectation value of $D_{0}$ assuming this frequency function, covering the entire range of $D_{0}$ values, is then

$$
\bar{D}_{0}(D)=\int_{0}^{\infty} D_{0} \varphi_{D_{0}} \mathrm{~d} D_{0}=\frac{1}{N(D)} \int_{0}^{\infty} D_{0} N_{D_{0}} e^{-D / D_{0}} \mathrm{~d} D_{0}
$$

and in the two-compartment case of the r-s model from equation (3.36) and paper IV,

$$
\bar{D}_{0}(D)=\frac{D_{\mathrm{r}} N_{\mathrm{r}} e^{-D / D_{\mathrm{r}}}+D_{\mathrm{s}} N_{\mathrm{s}} e^{-D / D_{\mathrm{s}}}}{N_{\mathrm{r}} e^{-D / D_{\mathrm{r}}}+N_{\mathrm{s}} e^{-D / D_{\mathrm{s}}}} .
$$

This can be compared to the two-compartment $D_{0, \text { eff }}$ defined in equation (3.38). Equation (3.38) can be written as

$$
D_{0, \text { eff }}=\frac{D_{\mathrm{s}} D_{\mathrm{r}} N_{\mathrm{r}} e^{-D / D_{\mathrm{r}}}+D_{\mathrm{r}} D_{\mathrm{s}} N_{\mathrm{s}} e^{-D / D_{\mathrm{s}}}}{D_{\mathrm{s}} N_{\mathrm{r}} e^{-D / D_{\mathrm{r}}}+D_{\mathrm{r}} N_{\mathrm{s}} e^{-D / D_{\mathrm{s}}}}
$$

showing that, for $D_{0 \text {,eff }}$ compared to $\bar{D}_{0}$, the resistant component is reduced by a factor of $D_{\mathrm{s}}$, while the sensitive compartment is increased by $D_{\mathrm{r}}$, since $D_{\mathrm{r}}>D_{\mathrm{s}}$.

The mean clonogen number $\bar{N}_{0}$, corresponding to $\bar{D}_{0}$ in equation $(3.51)$, can be found from $N(D)=\bar{N}_{0} e^{-D / \bar{D}_{0}}, c f$. equation (3.28), so that

$$
\bar{N}_{0}=N(D) e^{D / \bar{D}_{0}}=\left(\int N_{D_{0}} e^{-D / D_{0}} \mathrm{~d} D_{0}\right) \exp \left(\frac{\int D N_{D_{0}} e^{-D / D_{0}} \mathrm{~d} D_{0}}{\int D_{0} N_{D_{0}} e^{-D / D_{0}} \mathrm{~d} D_{0}}\right)
$$

and for two compartments

$$
\bar{N}_{0}=\left(N_{\mathrm{r}} e^{-D / D_{\mathrm{r}}}+N_{\mathrm{s}} e^{-D / D_{\mathrm{s}}}\right) \exp \left(\frac{D N_{\mathrm{r}} e^{-D / D_{\mathrm{r}}}+D N_{\mathrm{s}} e^{-D / D_{\mathrm{s}}}}{D_{\mathrm{r}} N_{\mathrm{r}} e^{-D / D_{\mathrm{r}}}+D_{\mathrm{s}} N_{\mathrm{s}} e^{-D / D_{\mathrm{s}}}}\right) .
$$

The expectation value in equation (3.51) is equivalent to the arithmetic mean value of $D_{0}$. The effective $D_{0}$ on the other hand, $c f$. equation (3.27), can be expressed as

$$
\frac{1}{D_{0, \mathrm{eff}}(D)}=\int \frac{\varphi_{D_{0}}}{D_{0}} \mathrm{~d} D_{0}
$$

which gives the harmonic mean value of $D_{0}$. See also the harmonic radiation resistance in relation to the $\mathrm{r}-\mathrm{s}$ model in equation (3.43) and paper IV. The $D_{0, \text { eff }}$ described in paper III is thus the harmonic mean radiation resistance corresponding to the frequency function $\varphi_{D_{0}}$. The parameter pair $\left\{D_{0, \text { eff }}, N_{0, \text { eff }}\right\}, c f$. equations (3.27) to (3.39), will always produce the best approximation to the true dose-response curve close to the 
dose $D$ where it is defined. A harmonic mean value is however always lower than the corresponding arithmetic mean. Hence, the arithmetic mean parameter pair $\left\{\bar{D}_{0}, \bar{N}_{0}\right\}$ presented in equations (3.51) to (3.55) will give a better approximation at doses above the dose level where the mean or effective values are defined. The best approximation near that dose level will however be achieved through the harmonic mean effective values, as defined in papers II and III.

In figure 3.2, the effective value approximation (the harmonic mean) is shown along with the arithmetic mean response, both evaluated at $D_{37}$. As stated above, the arithmetic mean indeed gives a better approximation to both the true survival curve and the tumor cure probability at doses above $D_{37}$. At lower doses, the effective value approximation is better. Both $\bar{D}_{0}$ (the slope of the survival curve) and $\bar{N}_{0}$ (the initial value of the survival curve) are lower than their effective counterparts.

The effective and mean values discussed here are weighted moments of $N_{D_{0}}, c f$. papers II, III and IV. In this context it may also be desirable to study the effective or mean values relevant over a certain dose or $D_{0}$ interval such as

$$
\bar{D}_{0,1,2}=\frac{\int_{D_{0,1}}^{D_{0,2}} D_{0} N_{D_{0}} e^{-D / D_{0}} \mathrm{~d} D_{0}}{\int_{D_{0,1}}^{D_{0,2}} N_{D_{0}} \mathrm{~d} D_{0}} .
$$

It can be seen from the weighting factor, $D_{0}$ compared to $1 / D_{0}$, that this kind of moment, in particular $\bar{D}_{0,0, \infty}$ in equation (3.51), is valid mainly at very high doses. This is because the $D_{0}$ weighting factor mainly considers the resistant clonogen population with high $D_{0}$ values, while the factor $1 / D_{0}$ in equation (3.51) gives a higher weight to the sensitive clonogens.

\subsection{Incorporating tumor dynamics in constant-dose fractionation modeling}

During the course of radiation therapy, both the distribution of radiation sensitivity and the number of clonogens will change due to factors such as cell kill, reoxygenation, proliferation and selection towards more resistant phenotypes. Such complex concepts are difficult to incorporate in cell survival modeling, and they are also difficult to estimate in treatment planning, especially for the individual patient. For simplified cases it may however be possible to include them, and we will give some examples. For two compartments, a similar approach was used by Wouters and Brown (1997). In clinical dose-response data, all dynamic processes relevant to treatment outcome are already implicitly included, as discussed in paper IV.

\subsubsection{No reoxygenation}

As a first approximation, the only thing affecting the sensitivity distribution can be assumed to be cell kill due to radiation, without directly accounting for reoxygenation 


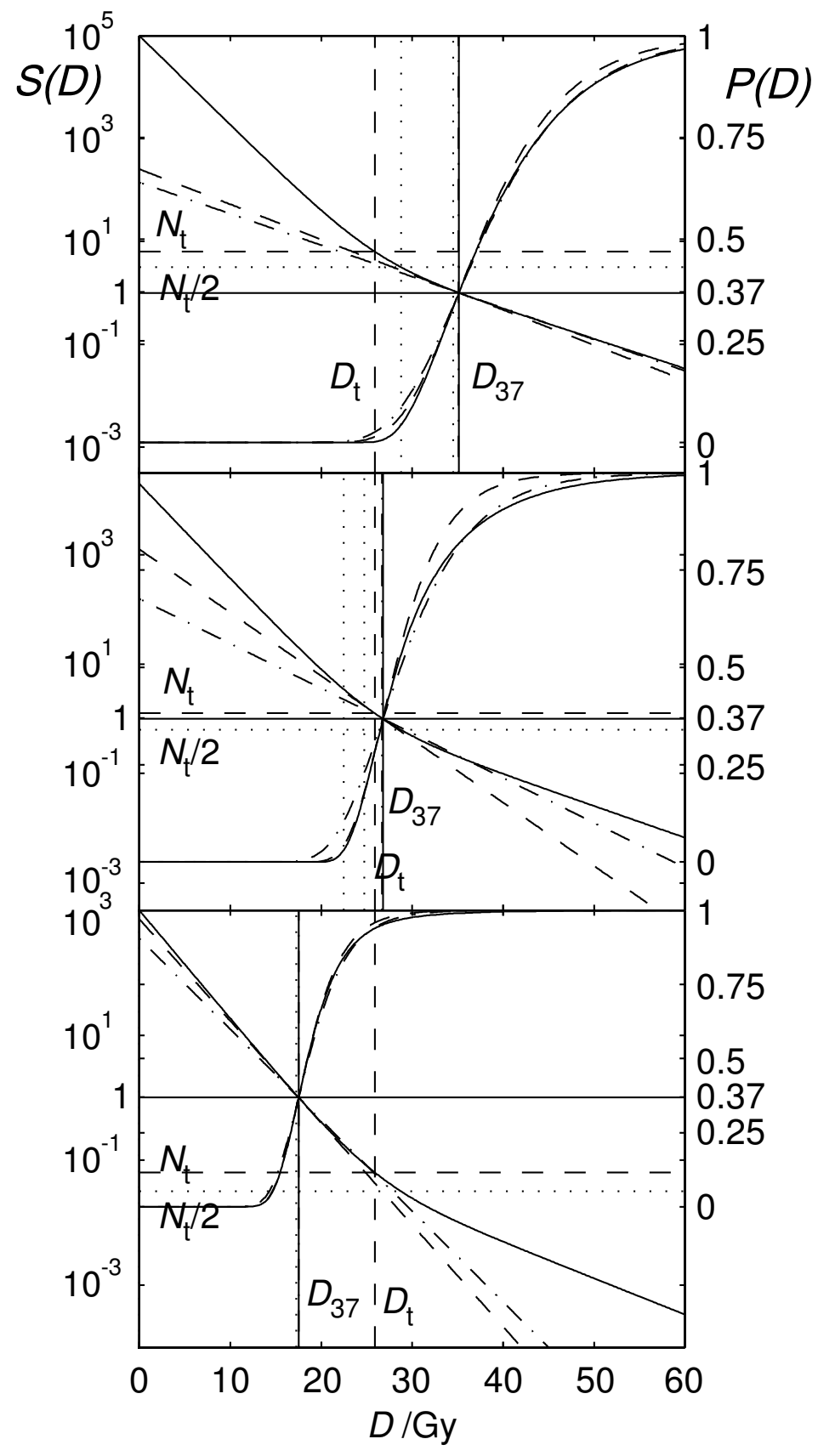

Figure 3.2.: Clonogen survival and dose-response relations for the three tumor types defined in paper IV. Vertical dotted lines show the individual $D_{37}$ values, $D_{37, \mathrm{~s}}$ and $D_{37, \mathrm{r}}$. The effective value and arithmetic mean approximations evaluated at $D_{37}$ are shown with dashed and dashed-dotted lines, respectively, $c f$. sections 3.6.2 and 3.7. The r-s model, equation (3.36), is used in all cases, with $D_{\mathrm{s}}=2.5 \mathrm{~Gy}, D_{\mathrm{r}}=7.5 \mathrm{~Gy}$ and $\nu=10^{-3}$. The total number of clonogens is, from top to bottom, $10^{5}, 2 \cdot 10^{4}$ and $10^{3}$, respectively. 
and proliferation. The distribution of surviving clonogens after fraction $i$ is then

$$
N_{D_{0}, i+1}=N_{D_{0}, i} S_{d}
$$

where $N_{D_{0}}$ is the distribution of radiation resistance, $c f$. paper III, and $S_{d}$ is the singledose clonogen survival probability, expressed as $S(d)$ in equation (3.7) and paper IV. For $n$ fractions, the behavior is similar to the simple case of uniform sensitivity, i.e.

$$
N_{D_{0}, n}=N_{D_{0}} \prod_{i=1}^{n} S_{d}=N_{D_{0}} S_{d}^{n}
$$

where $n=D / d, c f$. equation (3.7). The number of surviving clonogens is then

$$
N(D)=\int N_{D_{0}} S_{d}^{n} \mathrm{~d} D_{0}
$$

A mean general survival function, $\bar{S}(D)$, can in this case be defined by

$$
\bar{S}\left(D, D_{0}\right)=\frac{N(D)}{N_{0}}=\frac{\int N_{D_{0}} S_{d}^{n} \mathrm{~d} D_{0}}{\int N_{D_{0}} \mathrm{~d} D_{0}}
$$

\subsubsection{Reoxygenation}

A more complex case is when the distribution of surviving clonogens is reoxygenated into the original distribution in-between fractions, i.e. the distribution $N_{D_{0}, i+1}$ falls back to $N_{D_{0}, i}$, albeit with a smaller total number of clonogens. The distribution of surviving clonogens directly after irradiation then follows the same behavior as before, described by equation (3.58). Before the next treatment fraction however, assuming that proliferation does not occur, the distribution has changed back to the original one, only renormalized into the number of surviving clonogens, yielding

$$
N_{D_{0}, i+1}=N_{D_{0}, i} \frac{\int N_{D_{0}, i+1} \mathrm{~d} D_{0}}{\int N_{D_{0}, i} \mathrm{~d} D_{0}}=N_{D_{0}, i} \frac{\int N_{D_{0}, i} S_{d} \mathrm{~d} D_{0}}{\int N_{D_{0}, i} \mathrm{~d} D_{0}} .
$$

After $n$ fractions, it follows that

$$
N_{D_{0}, n}=N_{D_{0}}\left(\frac{\int N_{D_{0}} S_{d} \mathrm{~d} \chi}{\int N_{D_{0}} \mathrm{~d} \chi}\right)^{n} .
$$

The number of surviving clonogens is then

$$
N(D)=\int N_{D_{0}, n} \mathrm{~d} D_{0}=\left(\frac{\int N_{D_{0}} S_{d} \mathrm{~d} D_{0}}{\int N_{D_{0}} \mathrm{~d} D_{0}}\right)^{n} \int N_{D_{0}} \mathrm{~d} D_{0}=N_{0}^{1-n}\left(\int N_{D_{0}} S_{d} \mathrm{~d} D_{0}\right)^{n},
$$

where $N_{0}=\int N_{D_{0}} \mathrm{~d} D_{0}$. The mean general survival function, cf equation (3.61), becomes in this case

$$
\bar{S}\left(D, D_{0}\right)=\left(\frac{\int N_{D_{0}} S_{d} \mathrm{~d} D_{0}}{\int N_{D_{0}} \mathrm{~d} D_{0}}\right)^{n} .
$$


Accurate description of heterogeneous tumors

\subsubsection{Repopulation}

Tumor repopulation can be handled in response models for fractionated irradiation by multiplying $N_{0}$ with a factor that accounts for the accelerated repopulation that is often seen during radiation therapy. The factor $f_{i \text {,acc }}$ can be defined according to

$$
f_{i, \text { acc }}=\left\{\begin{array}{cl}
\exp \left(\frac{t_{i}-t_{i-1}}{T_{\mathrm{pot}}} \ln 2\right) & \text { if } t_{i}>T_{k} \\
1 & \text { if } t_{i} \leq T_{k}
\end{array},\right.
$$

where $t_{i}$ is the time when fraction $i$ is delivered, $T_{k}$ is the time of onset of accelerated proliferation and $T_{\text {pot }}$ is the potential doubling time of the tumor. The number of surviving clonogens based on a distribution of radiation resistance (see section 3.2) can be calculated using the method described by Deasy (1996). Simply multiplying the resistance distribution $N_{D_{0}}$ with $f_{i \text {,acc }}$ may not be accurate, as discussed for homogeneous populations by Tucker et al. (1990); Tucker and Taylor (1996). It is likely that repopulation occurs at different rates depending on, for example, the level of oxygenation. The repopulation factor $f_{i \text {,acc }}$ can be extended to include a $D_{0}$-dependence, which could then account for the oxygenation dependence.

\subsection{Oxygen dependence of the cell survival model parameters}

In paper IV, the LET-dependence of the LQ-model parameters $\alpha$ and $\beta$ was studied. A similar approach may be applied to the oxygen dependence of the survival model parameters. As was discussed for the LET dependence in paper IV, the OER can then be calculated for any survival level if needed.

The radiation-dose dependent survival of bacteria at different levels of oxygen concentration was studied by Alper and Howard-Flanders (1956). They established a semi-empirical relation between the increase in sensitivity with increasing oxygen concentration, which is often referred to as the Alper-Howard-Flanders equation, or the Alper $K$-curve. Alper (1979) later derived the relation based on Michaelis-Menten kinetics for biochemical reactions. The ratio of doses to achieve the same cell survival under oxic and anoxic conditions can thus be described as

$$
r=\frac{D_{\text {anoxic }}}{D_{\text {oxic }}}=\frac{m p_{\mathrm{O}_{2}}+K}{p_{\mathrm{O}_{2}}+K},
$$

where $D_{\text {anoxic }}$ and $D_{\text {oxic }}$ are the doses to achieve the same cell survival probability $S$, $p_{\mathrm{O}_{2}}$ is the oxygen partial pressure, $m$ is the maximum ratio and $K$ is the $p_{\mathrm{O}_{2}}$ at half the increase from unity to $m$. Applying the exponential cell survival probability described by equation (3.2), $D$ can be expressed as $D=-D_{0} \ln S$. The $p_{\mathrm{O}_{2}}$-dependent radiation resistance $D_{0}$ then becomes

$$
D_{0}\left(p_{\mathrm{O}_{2}}\right)=D_{0, \text { anoxic }} \frac{p_{\mathrm{O}_{2}}+K}{m p_{\mathrm{O}_{2}}+K}
$$


where $D_{0, \text { anoxic }}$ is the radiation resistance of viable clonogenic cells at zero $p_{\mathrm{O}_{2}}$. This latter expression was used in papers II, III and IV to calculate the radiation resistance distribution from a distribution in $p_{\mathrm{O}_{2}}$. The Alper-Howard-Flanders model has been extended to include $e$.g. the ratio of repairable to irrepairable damaged targets (Koch et al., 1984; Scott et al., 1993).

The oxygen dependence of the LQ model parameters has been studied by for example Wouters and Brown (1997) and Buffa et al. (2001), applying an OER to the $\alpha$ and $\beta$ parameters.
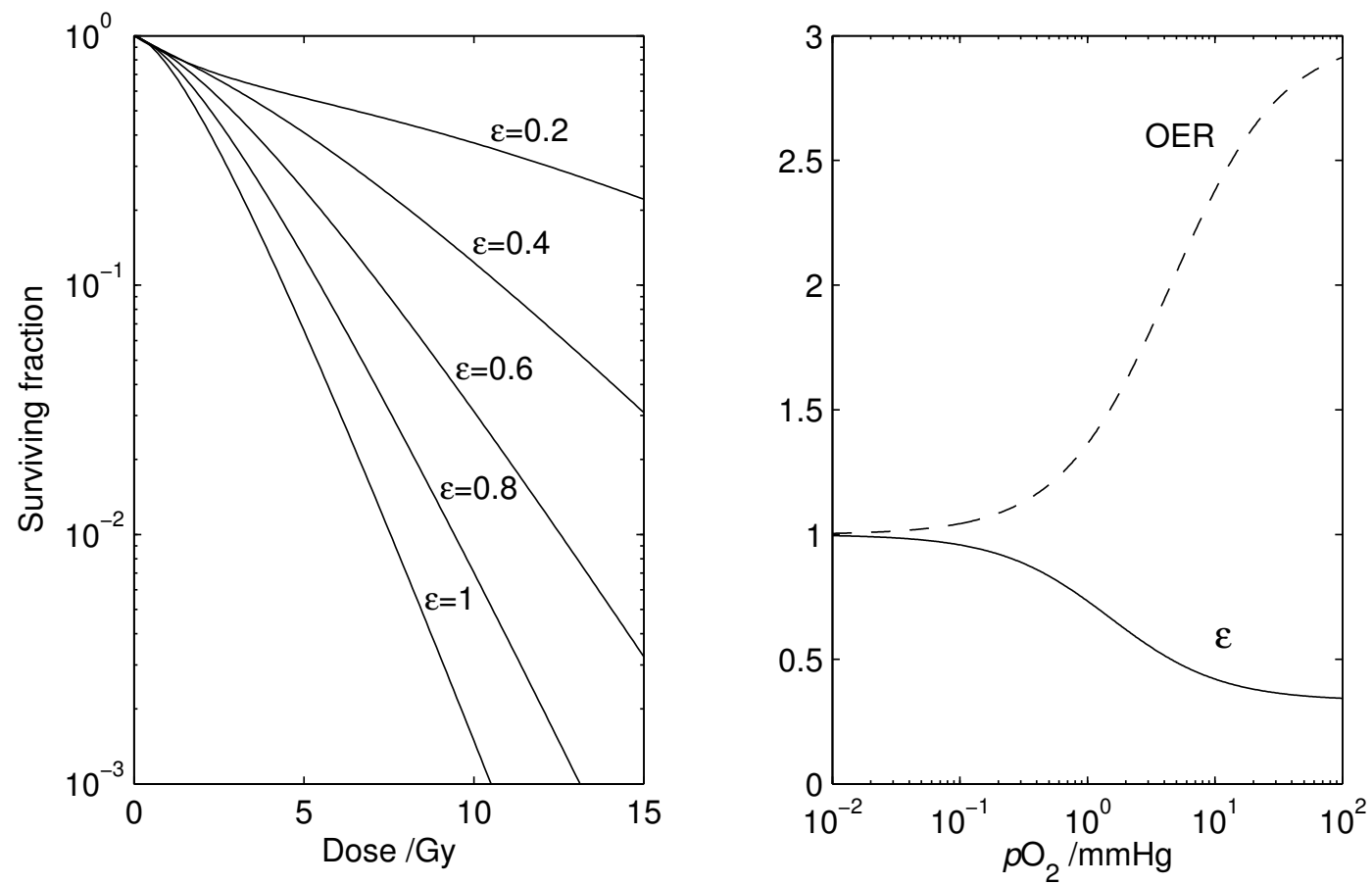

Figure 3.3.: Left panel: The RCR model for different values of $\varepsilon$. RCR model parameters from Persson (2002) for the M059K cell line: $a=1.11 \mathrm{~Gy}^{-1}, b=1.04$ $\mathrm{Gy}^{-1}, c=0.886 \mathrm{~Gy}^{-1}$ Right panel: The $\varepsilon=1 / \mathrm{OER}$ and OER as functions of $p_{\mathrm{O}_{2}}$ based on equation (3.68).

In a recent $\mathrm{PhD}$ thesis, Persson (2002) assumed that the amount of potentially repairable damage in the RCR model, equation (3.6), is reduced by a factor $\varepsilon$ during hypoxia. Under hypoxic conditions, the survival model parameter $c_{\text {hypoxia }}$ corresponding to $1 / D_{0}$ then becomes $\varepsilon c_{\text {air }}$. If the amount of conditionally repairable damage is constant, then $a_{\text {hypoxia }}=a_{\text {air }}-c_{\text {air }}(1-\varepsilon)$. The relative amount of potentially repairable damage that is actually repaired is assumed to be constant, and thus $b_{\text {hypoxia }}=\varepsilon b_{\text {air }}$. Based on these assumptions, the RCR model then takes the form

$$
S(D)=e^{-\left(a_{\text {air }}-c_{\text {air }}(1-\varepsilon)\right) D}+\varepsilon b_{\text {air }} D e^{-\varepsilon c_{\text {air }} D} .
$$


Notice that since $c$ corresponds to $1 / D_{0}$, and $c_{\text {hypoxia }}=\varepsilon c_{\text {air }}$ under hypoxic conditions, we have that $\varepsilon=c_{\text {hypoxia }} / c_{\text {air }}$. If OER $=D_{0 \text {,hypoxic }} / D_{0, \text { oxic }}, c f$. equation (3.68), it turns out that $\varepsilon \approx 1 / \mathrm{OER}$. Since the OER is limited to maximum values of around 3 to $4, \varepsilon$ has a lower limit around 0.25-0.33.

Figure 3.3 shows RCR survival curves for different values of $\varepsilon$, based on RCR parameters for the M059K cell line (Persson, 2002). The figure also shows $\varepsilon$ and the OER, as described by equation (3.67), as functions of $p_{\mathrm{O}_{2}}$.

\subsection{The response of hypoxic tumors to beams of low and high ionization density}

To express the influence on the clonogen radiation response, the ratio of iso-effective doses is often used. Relations such as RBE or OER are examples of such ratios, where the RBE describes the ratio of the efficiency of different radiation qualities on a given cell line and the OER describes the dose increase required of a given radiation modality for iso-effect in the presence or absence of oxygen. Both the RBE and OER are generally dependent on the chosen level of survival, and thus indirectly on the dose level. In the high dose region beyond the shoulder of the survival curve, cell survival is generally exponential, see section 3.1. If the RCR model is used, see equation (3.6), the dependence on the chosen survival level then disappears.

In biological treatment optimization, the whole range of doses from zero to the dose maximum have to be considered for different types of cells, tumors and healthy tissues. The simple RBE and OER relations are then impractical. A more general approach would be to introduce dependencies of $e . g \cdot p_{\mathrm{O}_{2}}$ and LET on all parameters in the survival model, as was discussed in section 3.9 and paper IV. Explicit values of RBE and OER can then be easily calculated for any survival level if needed.

In the r-s model, $c f$. equation (3.37) and paper IV, the radiation resistance parameters for the two compartments, $D_{\mathrm{r}}$ and $D_{\mathrm{s}}$, can be extracted from single-dose survival curves obtained under e.g. oxic and hypoxic conditions. Such a method was used in paper IV for human salivary gland (HSG) tumor cells irradiated with carbon ion beams, based on the data from Furusawa et al. (2000). While calculating the corresponding radiation resistance of a tumor with effective numbers of clonogens $N_{\mathrm{r}}$ and $N_{\mathrm{s}}$, the OER and RBE were not explicitly used. Instead, $D_{\mathrm{r}}$ and $D_{\mathrm{s}}$ were directly calculated based on the $\alpha$ and $\beta$ values as functions of the LET for HSG cells and ${ }^{12} \mathrm{C}$ published by Furusawa et al. (2000). 


\section{Conclusions}

A simple method for the description of tissue oxygenation heterogeneities has been developed. The model agrees very well with clinically observed oxygen distributions from a wide spectrum of normal healthy tissues and tumors. It is shown that the vessel density alone may not accurately describe the oxygenation of a given tissue, unless complemented with information about the heterogeneity of the vascular system.

The dose-response relation for a generally heterogeneous tumor can be well described by a single clonogen compartment, characterized by the effective radiation resistance and the effective clonogen number. This result is of great value for biologicallybased treatment optimization, since the computation time of the optimization procedure can be extensively reduced.

It is also shown how tumor oxygenation data from for example Eppendorf electrodes can be used to estimate the distribution of radiation resistance in the tumor and from that information quantify the expected tumor dose-response relation and how to best treat the tumor.

The normalized dose-response gradient has been generalized to take heterogeneities in both dose delivery and radiation response into account. This is an important contribution to the fast optimization of modern radiation therapy, and a valuable tool in analyzing treatment results.

For heterogeneous tumors, a two-compartment model of the radiation responsiveness has been developed and analyzed. The model describes the response of a heterogeneous tumor very well, both at low and high doses and LET values, if effective parameter values are used that are averaged over the whole spectrum of radiation sensitivity. This substantially simplifies biologically based treatment optimization of heterogeneous tumors, since only one or two different target cell compartments are needed rather than a whole continuum of cellular sensitivities.

In summary, methods are presented in this thesis that take distributions of radiation sensitivity into account in the prediction, optimization and analysis of radiation therapy outcome. It is shown that the dose-response relationship corresponding to such distributions can be accurately described using the effective resistant and sensitive cell compartments of heterogeneous tumors. 


\section{A. Appendix}

\section{A.1. Effective response parameters for some survival functions}

The effective parameters $D_{0, \text { eff }}$ and $N_{0, \text { eff }}$ for exponential cell survival, equation (3.4), were derived in papers II and III, see also section 3.4.1 and paper IV. Effective survival parameters can also be derived for more complex cell survival models.

\section{A.1.1. The LQ model}

An extensively used cell survival model is the LQ model, $c f$. section 3.1. Adapting the general survival function to this model yields, using the notation from section 3.3, $\vec{\chi}=\{\alpha, \beta\}$ and $S=S(\alpha, \beta, D)$, where the parameters $\alpha$ and $\beta$ are dependent on the variable $\chi$. The effective values of $\alpha, \beta$ and $N_{0}$ defined at the dose level $D$ then become

$$
\begin{gathered}
\alpha_{\mathrm{eff}}=\frac{1}{N^{2}}\left[N\left(N^{\prime \prime}-N^{\prime}\right)-\left(N^{\prime}\right)^{2}\right], \\
\beta_{\mathrm{eff}}=\frac{1}{2 D N^{2}}\left[\left(N^{\prime}\right)^{2}-N N^{\prime \prime}\right]
\end{gathered}
$$

and

$$
N_{0, \mathrm{eff}}=N e^{\alpha_{\mathrm{eff}} D+\beta_{\mathrm{eff}} D^{2}}=N \exp \left[\frac{D}{2 N^{2}}\left(N N^{\prime \prime}-2 N N^{\prime}-\left(N^{\prime}\right)^{2}\right)\right]
$$

where

$$
\begin{gathered}
N=\int N_{\chi} e^{-\alpha D-\beta D^{2}} \mathrm{~d} \chi \\
N^{\prime}=\frac{\partial N}{\partial D}=-\int N_{\chi}(\alpha+2 \beta D) e^{-\alpha D-\beta D^{2}} \mathrm{~d} \chi \\
N^{\prime \prime}=\frac{\partial^{2} N}{\partial D^{2}}=\int N_{\chi}\left[(\alpha+2 \beta D)^{2}-2 \beta\right] e^{-\alpha D-\beta D^{2}} \mathrm{~d} \chi .
\end{gathered}
$$




\section{A.1.2. The RCR model}

To take into account the fine details of cell survival such as low dose hypersensitivity, the RCR (Repairable-Conditionally-Repairable) model was recently developed by Lind et al. (2003), see section 3.1. In this model, three parameters are used to more accurately describe the cellular radiation response at low, intermediate and high doses through $S(a, b, c, D)=e^{-a D}+b D e^{-c D}$. Calculation of the effective values for the RCR model, in the same way as for the LQ model above, unfortunately leads to transcendental equations, and is therefore not possible analytically. However, it is possible semi-analytically or by using equations (A.1) to (A.3) to calculate the effective linearquadratic response corresponding to distributions in one variable in the RCR model parameters, while using the relations

$$
\begin{gathered}
N=\int N_{\chi}\left(e^{-a D}+b D e^{-c D}\right) \mathrm{d} \chi \\
N^{\prime}=\int N_{\chi}\left[-a e^{-a D}+b(1-c D) e^{-c D}\right] \mathrm{d} \chi
\end{gathered}
$$

and

$$
N^{\prime \prime}=\int N_{\chi}\left[a^{2} e^{-a D}-b c(2-c D) e^{-c D}\right] \mathrm{d} \chi,
$$

see also Fredriksson (2002).

The model parameter $c$ is related to exponential survival, equation (3.2), through $c \approx 1 / D_{0}$. At low doses, the RCR model can be expressed in a linear-quadratic fashion according to

$$
\begin{aligned}
S(a, b, c, D)=e^{-(a-b) D}\left(e^{-b D}\right. & \left.+b D e^{-(b+c-a) D}\right) \approx \\
& \approx \exp \left[-(a-b) D-\left(\frac{b}{2}+c-a\right) b D^{2}\right]
\end{aligned}
$$

see Lind et al. (2003) and Fredriksson (2002). For this expression, the effective response parameters for the LQ model from section A.1.1 may be used, with $\alpha=a-b$ and $\beta=b / 2+c-a$.

\section{A.2. The effective radiation response in terms of $D_{0, \text { eff }}$ and $N_{0, \text { eff }}$}

Similarly, the number of surviving cells of any survival model can be approximated by exponential survival, $c f$. papers II and III, and section 3.3:

$$
N(D)=\int N_{\chi} S(\chi, D) \mathrm{d} \chi \approx N_{0, \mathrm{eff}} e^{-D / D_{0, \mathrm{eff}}}
$$


The first-order derivative of this relation is

$$
\frac{\partial N(D)}{\partial D}=\int N_{\chi} S^{\prime}(\chi, D) \mathrm{d} \chi \approx-\frac{N_{0, \mathrm{eff}}}{D_{0, \mathrm{eff}}} e^{-D / D_{0, \mathrm{eff}}}
$$

At the dose level $D$ where the relations (A.11) and (A.12) are exact, the effective values are thus given by the ratio of (A.11) and (A.12):

$$
D_{0, \mathrm{eff}}=-\frac{\int N_{\chi} S(\chi, D) \mathrm{d} \chi}{\int N_{\chi} S^{\prime}(\chi, D) \mathrm{d} \chi}
$$

and

$$
N_{0, \mathrm{eff}}=N(D) e^{D / D_{0, \mathrm{eff}}}=\left[\int N_{\chi} S(\chi, D) \mathrm{d} \chi\right] \exp \left(-D \frac{\int N_{\chi} S^{\prime}(\chi, D) \mathrm{d} \chi}{\int N_{\chi} S(\chi, D) \mathrm{d} \chi}\right) .
$$

\section{A.2.1. The LQ model}

The effective exponential cell survival for the LQ model is given by equations (A.13) and (A.14) together with (A.5):

$$
D_{0, \mathrm{eff}}=\frac{\int N_{\chi} e^{-\alpha D-\beta D^{2}} \mathrm{~d} \chi}{\int N_{\chi}(\alpha+2 \beta D) e^{-\alpha D-\beta D^{2}} \mathrm{~d} \chi}
$$

and

$$
N_{0, \mathrm{eff}}=\left[\int N_{\chi} e^{-\alpha D-\beta D^{2}} \mathrm{~d} \chi\right] \exp \left(\frac{\int N_{\chi} D(\alpha+2 \beta D) e^{-\alpha D-\beta D^{2}} \mathrm{~d} \chi}{\int N_{\chi} e^{-\alpha D-\beta D^{2}} \mathrm{~d} \chi}\right) .
$$

\section{A.2.2. The RCR model}

Calculating the purely exponential effective values for the RCR model using equations (A.13), (A.14) and (A.8) generates

$$
D_{0, \text { eff }}=\frac{\int N_{\chi}\left(e^{-a D}+b D e^{-c D}\right) \mathrm{d} \chi}{\int N_{\chi}\left[a e^{-a D}+b(c D-1) e^{-c D}\right] \mathrm{d} \chi}
$$

and

$$
N_{0, \mathrm{eff}}=\left[\int N_{\chi}\left(e^{-a D}+b D e^{-c D}\right) \mathrm{d} \chi\right] \exp \left(\frac{\int N_{\chi} D\left(a e^{-a D}+b(c D-1) e^{-c D}\right) \mathrm{d} \chi}{\int N_{\chi}\left(e^{-a D}+b D e^{-c D}\right) \mathrm{d} \chi}\right) .
$$




\section{Acknowledgments}

I would like to express my sincere gratitude to all the people that have assisted me on my path towards finishing this thesis. Among many others, thanks to:

Anders Brahme, for your scientific guidance and your fountain of visions and ideas, and for showing a constant interest in my work.

Bengt Lind, my main supervisor, for support and encouragement, and for your active listening.

Margareta Edgren for sharing your great knowledge about radiobiology. Dževad Belkić for help with mathematics and good advice. Malin Hollmark for constant support, and for sharing some of the ups and downs of life. Brigida da Costa Ferreira, for your great encouragement and support. Sara Janek. Sharif Qatarneh for good companionship, not only while representing the research students. Magdalena Adamus-Górka for great roommate-ship, and for making our office less hypoxic. Björn Skatt, my former roommate, for nice chatting about many things, and for assistance in numerical analysis. Linda Persson, another former roommate, for nice chatting and support in the early days. Olof Sjörs, my early-days server-room roommate, for good companionship both in vivo and in silico. Bo Nilsson for your great dedication and patience in trying to teach us medical radiation physics. Lil, Ann-Charlotte and coworkers for, among other things, administrative support.

Thanks also to all others at MSF for creating a nice environment.

Tack även till min pappa Ingemar och min bror Patrik och Marina. Till min mamma Eva, som såg mig som humanist men upptäckte att jag nog kunde klara en del naturvetenskap också.

Tack Ingrid och Leo, för all kärlek och allt stöd. Tack för att ni gör min framtid så ljus.

This work was partially supported by the King Gustaf V's Jubilee Fund, the Cancer Society in Stockholm and the Research Center for Radiation Therapy, VINNOVA Center of Excellence at Karolinska Institutet. 


\section{Bibliography}

Ågren-Cronqvist, A., Källman, P., Turesson, I. and Brahme, A., 1995, Volume and heterogeneity dependence of the dose-response relationship for head and neck tumours, Acta Oncologica, 34, 851-860.

AlPER, T., 1979, Cellular Radiobiology (Cambridge: Cambridge University Press).

Alper, T. and HowARD-Flanders, P., 1956, The role of oxygen in modifying the radiosensitivity of $E$. coli B., Nature, 178, 978-979.

Awwad, H. K., el Naggar, M., Mocktar, N. and Barsoum, M., 1986, Intercapillary distance measurement as an indicator of hypoxia in carcinoma of the cervix uteri, International Journal of Radiation Oncology Biology and Physics, 12 , 1329-1333.

Baish, J. W., Gazit, Y., Berk, D. A., Nozue, M., Baxter, L. T. and Jain, R. K., 1996, Role of tumor vascular architecture in nutrient and drug delivery: an invasion percolation-based network model, Microvascular Research, 51, 327-346.

Bentzen, S. M. and Thames, H. D., 1996, Tumor volume and local control probability: clinical data and radiobiological interpretations, International Journal of Radiation Oncology Biology and Physics, 36, 247-251.

Borkenstein, K., Levegrün, S. and Peschke, P., 2004, Modeling and computer simulations of tumor growth and tumor response to radiotherapy, Radiation research, 162, 71-83.

BrAhme, A., 1984, Dosimetric precision requirements in radiation therapy, Acta Radiologica Oncology, 23, 379-391.

Brahme, A. and ÅGREn, A. K., 1987, Optimal dose distribution for eradication of heterogeneous tumours, Acta Oncologica, 26, 377-385.

BRown, J. M., 1979, Evidence for acutely hypoxic cells in mouse tumours, and a possible mechanism of reoxygenation, British Journal of Radiology, 52, 650-656. 
Buffa, F. M., West, C., Byrne, K., Moore, J. V. and Nahum, A. E., 2001, Radiation response and cure rate of human colon adenocarcinoma spheroids of different size: The significance of hypoxia on tumor control modeling, International Journal of Radiation Oncology Biology and Physics, 49, 1109-1118.

Bussink, J., KaAnders, J. H., Rijken, P. F., Peters, J. P., Hodgkiss, R. J., Marres, H. A. and VAN Der Kogel, A. J., 1999, Vascular architecture and microenvironmental parameters in human squamous cell carcinoma xenografts: effects of carbogen and nicotinamide, Radiotherapy and Oncology, 50, 173-184.

CHADwick, K. H. and LeEnhouts, H. P., 1973, A molecular theory of cell survival, Physics in Medicine and Biology, 18, 78-87.

Chapman, J. D., 1979, Hypoxic sensitizers - implications for radiation therapy, $N$ Engl J Med, 301, 1429-1432.

Chapman, J. D., 1991, Measurement of tumor hypoxia by invasive and non-invasive procedures: a review of recent clinical studies, Radiotherapy and Oncology, 20, $13-19$.

Chapman, J. D., Engelhardt, E. L., Stobbe, C. C., Schneider, R. F. and HANKS, G. E., 1998, Measuring hypoxia and predicting tumor radioresistance with nuclear medicine assays, Radiotherapy and Oncology, 46, 229-237.

Chapman, J. D., Schneider, R. F., Urbain, J.-L. and Hanks, G. E., 2001, Single-photon emission computed tomography and positron-emission tomography assays for tissue oxygenation, Seminars in Radiation Oncology, 11, 47-57.

Collingridge, D. R., Young, W. K., Vojnovic, B., Wardman, P., Lynch, E. M., Hill, S. A. and Chapman, D. J., 1997, Measurement of tumour oxygenation: a comparison between polarographic needle electrodes and a time-resolved luminescence-based optical sensor, Radiation Research, 147, 329-334.

Cooper, R. A., Carrington, B. M., Loncaster, J. A., Todd, S. M., DavidSon, S. E., Logue, J. P., Luthra, A. D., Jones, A. P., Stratford, I., Hunter, R. D. and West, C. M. L., 2000, Tumour oxygenation levels correlate with dynamic contrast-enhanced magnetic resonance imaging parameters in carcinoma of the cervix, Radiotherapy and Oncology, 57, 53-59.

CURTIS, S. B., 1986, Lethal and potentially lethal lesions induced by radiation - a unified repair model, Radiation Research, 106, 252-270.

Daşu, A., Toma-Daşu, I. and Karlsson, M., 2003, Theoretical simulation of tumour oxygenation and results from acute and chronic hypoxia, Physics in Medicine and Biology, 48, 2829-2842. 
DEASY, J., 1996, Poisson formulas for tumor control probability with clonogen proliferation, Radiation Research, 145, 382-384.

DELIDES, G. S., VENIZElos, J. and RÉVÉSZ, L., 1988, Vascularization and curability of stage III and IV nasopharyngeal tumors, J Cancer Research Clin Oncol, 114, 321-323.

Douglas, B. G. and Fowler, J. F., 1975, Fractionation schedules and a quadratic dose-effect relationship, British Journal of Radiology, 48, 502-504.

Evans, S. M. and Koch, C. J., 2003, Prognostic significance of tumor oxygenation in humans, Cancer Letters, 195, 1-16.

FREDRIKSSON, M., 2002, Biological fractionation effects in radiation therapy optimization, M.Sc. thesis, Medical Radiation Physics, Karolinska Institutet and Stockholm University (Royal Institute of Technology, Stockholm, E254).

Furusawa, Y., Fukutsu, K., Aoki, M., Itsukaichi, H., Eguchi-Kasai, K., OHARA, H., YATAGAI, F., KANAI, T. and ANDO, K., 2000, Inactivation of aerobic and hypoxic cells from three different cell lines by accelerated ${ }^{3} \mathrm{He}-,{ }^{12} \mathrm{C}$ - and ${ }^{20} \mathrm{Ne}-$ ion beams, Radiation Research, 154, 485-496.

Gazit, Y., Baish, J. W., Safabakhsh, N., Leunig, M., BaXter, L. T. and Jain, R. K., 1997, Fractal characteristics of tumor vascular architecture during tumor growth and regression, Microcirculation, 4, 395-402.

Gazit, Y., Berk, D., Leunig, M., BaXter, L. T. and Jain, R. K., 1995, Scaleinvariant behavior and vascular network formation in normal and tumor tissue, Physical Review Letters, 75, 2428-2431.

Groebe, K. and Vaupel, P., 1988, Evaluation of oxygen diffusion distances in human breast cancer xenografts using tumor-specific in vivo data: role of various mechanisms in the development of tumor hypoxia, International Journal of Radiation Oncology Biology and Physics, 15, 691-697.

Grunewald, W. A. and Sowa, W., 1977, Capillary structures and $\mathrm{O}_{2}$ supply to tissue - an analysis with a digital diffusion model as applied to the skeletal muscle, Reviews in Physiology, Biochemistry and Pharmacology, 77, 149-209.

Höckel, M., Schlenger, K., Knoop, C. and VAupel, P., 1991, Oxygenation of carcinomas of the uterine cervix: evaluation by computerized $\mathrm{O} 2$ tension measurements, Cancer Research, 51, 6098-6102.

Hollmark, M., Uhrdin, J., Belkić, D., Gudowska, I. and Brahme, A., 2004, Influence of multiple scattering and energy loss straggling on the absorbed dose distributions of therapeutic light ion beams: I. Analytical pencil beam model, Physics in Medicine and Biology, 49, 3247-3265. 
HOPER, J. and JAHN, H., 1995, Influence of environmental oxygen concentration on growth and vascular density of the area vasculosa in chick embryos, Int J Microcirc Clin Exp, 15, 186-192.

Johnson, N. L., Kotz, S. and Kemp, A. W., 1993, Univariate Discrete Distributions (John Wiley \& Sons Inc.), 2nd edition.

Kallinowski, F., Zander, R., Höckel, M. and Vaupel, P., 1990, Tumor tissue oxygenation as evaluated by computerized pO2 histography, International Journal of Radiation Oncology Biology and Physics, 19, 953-961.

Källman, P., Ågren, A. and Brahme, A., 1992, Tumour and normal tissue responses to fractionated non-uniform dose delivery, International Journal of Radiation Biology, 62, 249-262.

Kellerer, A. M. and Hug, O., 1972, Theory of Dose-Effect Relationships (Springer-Verlag, Berlin and New York), volume 2.

Khalil, A. A., Bentzen, S. M. and OvergaArd, J., 1997, Steepness of the doseresponse curve as a function of volume in an experimental tumor irradiated under ambient or hypoxic conditions [see comments], International Journal of Radiation Oncology Biology and Physics, 39, 797-802.

Koch, C. J., Stobbe, C. C. and Bump, E. A., 1984, The effect on the $K_{m}$ for radiosensitization at $0^{\circ} \mathrm{C}$ of thiol depletion by diethylmaleate pretreatment: Quantitative differences found using the radiation sensitizing agent misonidazole or oxygen, Radiation Research, 98, 141-153.

Kocher, M. and Treuer, H., 1995, Reoxygenation of hypoxic cells by tumor shrinkage during irradiation. a computer simulation, Strahlenther Onkol, 171, 219230 .

Kocher, M., Treuer, H. and Muller, R. P., 1997, Quantification of tumor reoxygenation during accelerated radiation therapy, Radiology, 205, 263-268.

Koh, W. J., Rasey, J. S., Evans, M. L., Grierson, J. R., Lewellen, T. K., Graham, M. M., Krohn, K. A. and Griffin, T. W., 1991, Imaging of hypoxia in human tumors with [F-18]fluoromisonidazole, International Journal of Radiation Oncology Biology and Physics, 22, 199-212.

KolstaD, P., 1968, Intercapillary distance, oxygen tension and local recurrence in cervix cancer, Scand J Clin Lab Invest Suppl, 106, 145-157.

Konerding, M. A., Malkusch, W., Klapthor, B., van Ackern, C., Fait, E., Hill, S. A., Parkins, C., Chaplin, D. J., Presta, M. and Denekamp, J., 1999, Evidence for characteristic vascular patterns in solid tumors: quantitative studies using corrosion casts, British Journal of Cancer, 80, 724-732. 
Krishna, M. C., Subramanian, S., Kuppusamy, P. and Mitchell, J. B., 2001, Magnetic resonance imaging for in vivo assessment of tissue oxygen concentration, Seminars in Radiation Oncology, 11, 58-69.

KROGH, A., 1919, The number and distribution of capillaries in muscles with calculations of the oxygen pressure head necessary for supplying the tissue, Journal of Physiology (London), 52, 409-415.

Lauk, S., Skates, S., Goodman, M. and Suit, H. D., 1989, A morphometric study of the vascularity of oral squamous cell carcinomas and its relation to outcome of radiation therapy, European Journal of Cancer and Clinical Oncology, 25, 14311440 .

LinD, B. K. and BRAHME, A., 1997, The effect on tumor response due to inter-patient variability in sensitivity and clonogen number, in Proceedings of the XIIth ICCR.

Lind, B. K., Persson, L. M., Edgren, M. R., HedlöF, I. and Brahme, A., 2003, Repairable-Conditionally-Repairable damage model based on dual Poisson processes, Radiation Research, 160, 366-375.

LindBorG, L. and BRAhME, A., 1990, Influence of microdosimetric quantities on observed dose-response relationships in radiation therapy, Radiation Research, 124, S23-S28.

LöF, J., 2000, Development of a general framework for optimization of radiation therapy., Ph.D. thesis, Dept of Medical Radiation Physics, Stockholm University and Karolinska Institutet, Stockholm, Sweden.

Mankoff, D. A. and Bellon, J. R., 2001, Positron-emission tomographic imaging of cancer: Glucose metabolism and beyond, Seminars in Radiation Oncology, 11, $16-27$.

Marples, B., Lambin, P., Skov, K. A. and Joiner, M. C., 1997, Low dose hypersensitivity and increased radioresistance in mammalian cells, International Journal of Radiation Biology, 71, 721-735.

Marples, B., Wouters, B. G., Collins, S. J., Chalmers, A. J. and Joiner, M. C., 2004, Low-dose hyper-radiosensitivity: a consequence of ineffective cell cycle arrest of radiation-damaged $\mathrm{G}_{2}$-phase cells, Radiation Research, 161, 247255.

Munro, T. R. and Gilbert, C. W., 1961, The relation between tumour lethal doses and the radiosensitivity of tumour cells, British Journal of Radiology, 34, 246-251.

NiLsson, J., 1999, The dose-response relation for hypoxic tumors, M.Sc. thesis, Medical Radiation Physics, Karolinska Institutet and Stockholm University (Uppsala University, UPTEC F99041). 
Nordsmark, M., Bentzen, S. M. and OvergaARd, J., 1994, Measurement of human tumour oxygenation status by a polarographic needle electrode. an analysis of inter- and intratumour heterogeneity, Acta Oncologica, 33, 383-389.

PERSSON, L. M., 2002, Cell survival at low and high ionisation densities investigated with a new model, Ph.D. thesis, Dept of Medical Radiation Physics, Stockholm University and Karolinska Institutet, Stockholm, Sweden.

Plasswilm, L., Hoper, J., Cordes, N. and TAnnapfel, A., 1999, Investigation of microvessel density after irradiation, Radiation Research, 151, 454-460.

Pogue, B. W., O’hara, J. A., Wilmot, C. M., Paulsen, K. D. and Swartz, H. M., 2001, Estimation of oxygen distribution in RIF-1 tumors by diffusion modelbased interpretation of pimonidazole and Eppendorf measurements, Radiation Research, 155, 15-25.

Popel, A. S., 1989, Theory of oxygen transport to tissue, Critical reviews in biomedical engineering, 17, 257-321.

PORTER, E. H., 1980, The statistics of dose/cure relationships for irradiated tumors, British Journal of Radiology, 53, 210-227, 336-345, Parts I \& II.

Van Putten, L. M. and Kallman, R. F., 1968, Oxygenation status of a transplantable tumour during fractionated radiation therapy, Journal of the National Cancer Institute, 40, 441-451.

Rasey, J. S., Koh, W.-J., Evans, M. L., Peterson, L. M., Lewellen, T. K., Graham, M. M. and Krohn, K. A., 1996, Quantifying regional hypoxia in human tumors with positron emission tomography of $\left[{ }^{18} \mathrm{~F}\right]$ fluoromisonidazole: A pretherapy study of 37 patients, International Journal of Radiation Oncology Biology and Physics, 36, 417-428.

Révész, L., Siracka, E., Siracky, J., Delides, G. and PaVlaki, K., 1989, Variation of vascular density within and between tumors of the uterine cervix and its predictive value for radiotherapy, International Journal of Radiation Oncology Biology and Physics, 16, 1161-1163.

Scott, O. C. A., RÉvéSz, L. and Edgren, M., 1993, The 'X model': a modified version of the competition theory, International Journal of Radiation Biology, 64, 367-373.

Secomb, T. W., Hsu, R., Dewhirst, M. W., Klitzman, B. and Gross, J. F., 1993, Analysis of oxygen transport to tumor tissue by microvascular networks, International Journal of Radiation Oncology Biology and Physics, 25, 481-489.

Steel, G. G., McMillan, T. J. and Peacock, J. H., 1989, The 5 Rs of radiobiology, International Journal of Radiation Biology, 56, 1045-1048. 
Suit, H. D., ShaleK, R. J. and WetTe, R., 1965, Radiation response of C3H mammary carcinoma, in Cellular Radiation Biology (Williams and Wilkins, Baltimore), pp. 514-530.

TANNOCK, I. F., 1972, Oxygen diffusion and the distribution of cellular radiosensitivity in tumours, British Journal of Radiology, 45, 515-524.

Thomlinson, R. H. and Gray, L. H., 1955, The histological structure of some human lung cancers and the possible implications for radiotherapy, British Journal of Cancer, 9, 539-549.

Tilikidis, A. and Brahme, A., 1994, Microdosimetric description of beam quality and biological effectiveness in radiation therapy, Acta Oncologica, 33, 457-469.

Toma-Daşu, I., Daşu, A., Waites, A. and DeneKamp, J., 2001, Theoretical simulation of oxygen tension measurement in tissue using a microelectrode: I. The response function of the electrode, Physiological Measurement, 22, 713-725.

Toma-Daşu, I., Waites, A., Daşu, A., Denekamp, J. and Fowler, J. F., 2002, Theoretical simulation of oxygen tension measurement in tissue using a microelectrode: II. Simulated measurements in tissues, Radiotherapy and Oncology, 64, 109118.

TUCKER, S. L. and TAYLOR, J. M. G., 1996, Improved models of tumor cure, International Journal of Radiation Biology, 70, 539-553.

Tucker, S. L., Thames, H. D. and TAYlor, J. M. G., 1990, How well is the probability of tumor cure after fractionated irradiation described by Poisson statistics?, Radiation Research, 124, 273-282.

VAupel, P., Kallinowski, F. and OKUniefF, P., 1989, Blood flow, oxygen and nutrient supply, and metabolic microenvironment of human tumors: a review, Cancer Research, 49, 6449-6465.

Vaupel, P., Thews, O., Kelleher, D. K. and Hoeckel, M., 1998, Current status of knowledge and critical issues in tumor oxygenation. Results from 25 years of research in tumor pathophysiology, Advances in Experimental Medicine and Biology, 454, 591-602.

Withers, H. R., 1975, The four R's of radiotherapy, Advances in Radiation Biology, 5, 241-271.

Wouters, B. G. and Brown, J. M., 1997, Cells at intermediate oxygen levels can be more important than the "hypoxic fraction" in determining tumor response to fractionated radiotherapy, Radiation Research, 147, 541-550.

ZAMIR, M., 2001, Fractal dimensions and multifractility in vascular branching, Journal of Theoretical Biology, 212, 183-190. 